\title{
Estimation of Multicomponent Reliability Based on Progressively Type II Censored Data from Unit Weibull Distribution
}

\author{
Refah Mohammed Alotaibi ${ }^{1}$, Yogesh Mani Tripathi $^{2}$, SAnKu DeY $^{3}$, Hoda Ragab RezK ${ }^{1.4}$ \\ ${ }^{1}$ Mathematical Sciences Department, College of Science, Princess Nourah bint Abdulrahman University, \\ Riyadh, SAUDI ARABIA \\ ${ }^{2}$ Department of Mathematics, Indian Institute of Technology Patna, Bihta-801106, INDIA \\ ${ }^{3}$ Department of Statistics, St. Anthony's College, Shillong-793001, Meghalaya, INDIA \\ ${ }^{4}$ Department of statistics, Al-Azhar University, Cairo, EGYPT
}

\begin{abstract}
In this paper, inference upon stress-strength reliability is considered for unit-Weibull distributions with a common parameter under the assumption that data are observed using progressive type II censoring. We obtain di_erent estimators of system reliability using classical and Bayesian procedures. Asymptotic interval is constructed based on Fisher information matrix. Besides, boot-p and boot-t intervals are also obtained. We evaluate Bayes estimates using Lindley's technique and Metropolis-Hastings ( $\mathrm{MH}$ ) algorithm. The Bayes credible interval is evaluated using $\mathrm{MH}$ method. An unbiased estimator of this parametric function is also obtained under know common parameter case. Numerical simulations are performed to compare estimation methods. Finally, a data set is studied for illustration purposes.
\end{abstract}

Keywords: Bayes estimator; Multicomponent reliability; Progressive censoring; Maximum likelihood estimate; Uniformly minimum variance unbiased estimator.

Received: February 3, 2021. Revised: May 23, 2021. Accepted: June 15, 2021. Published: July 2, 2021.

\section{Introduction}

The element of uncertainty involved in various fields of study are often analyzed by means of various well known classical probability models like gamma family, Weibull, lognormal, Gompertz, Burr distributions, generalized exponential etc. Such models have positive probability over half real line. However, in many physical phenomena, we often encounter characteristics like average rainfall in a geographical area, mean and variance of individuals, score values of aptitude tests, control indices of a process which lie on bounded interval [see also [31]]. In recent years several new probability models with finite support have been introduced, see, [23], [28] and [29]. Thus it is of practical interest to consider probability distributions with finite support to derive better inferences for unknown quantities. Besides, it is significant to have models proposed on finite interval for plausible results, especially in case of measurement of reliability as percentage or ratio. Motivated by this rationale, [30] studied unit Weibull (UW) distribution and reported various properties.
The pdf of UW distribution is given by $f_{X}(x, \alpha, \beta)=\alpha \beta(-\ln x)^{\beta-1} x^{-1} e^{-\alpha(-\ln x)^{\beta}}, 0<x<1, \alpha>0, \beta>0$;

and cdf is

$$
F_{X}(x)=e^{-\alpha(-\ln x)^{\beta}}, 0<x<1, \alpha>0, \beta>0 .
$$

Here $\alpha$ and $\beta$ reflect shape parameters. From now on wards, we use the notation $U W(\alpha, \beta)$ for above model. Its density function can acquire various shapes such as unimodal and anti-unimodal, increasing or decreasing. The corresponding hazard rate is either constant or increasing or bathtub-shaped depending upon range of its parameters. This distribution is used as an alternative probability rule to many established distributions with positive support on finite range such as beta and Kumaraswamy distributions. Its percentile can be easily computed. It is to be noted that, if $\alpha=\beta=1$, the UW distribution gives uniform distribution with support on unit intervals $(0,1)$, if $\beta=1$, the UW distribution reduces to power function distribution while if $\beta=2$ the UW distribution produces to unit-Rayleigh distribution. Hence, it is obvious 
that the new model is inter related to established models and therefore it can be very useful in many studies.

In this paper, we evaluate various estimators of reliability based on unit Weibull distribution when data are observed using a given censoring. This progressive censoring is applied in various life test studies to derive important inferences. We briefly describe this scheme for better comprehension: Let $M$ systems each having $K$ components be subjected to a test. The corresponding data are derived as follows. When first failure occurs, then $g_{1}$ live items are removed from remaining active $M-1$ systems. At this stage $f_{1}$ components are also taken away from the active $K-1$ components. Similarly, when second observations occurs, $g_{2}$ systems are removed from the remaining active $M-g_{1}-2$ units. Also $f_{2}$ components are taken away from the active $K-f_{1}-2$ components. This sequence of observations stops at $m$ th failure and by this the failure times of $k$ components are recorded. The remaining active items $g_{m}=M-m-g_{1}-\ldots-g_{m-1}$ are withdrawn along with $f_{k}=K-k-f_{1}-\ldots-f_{k-1}$ components from life testing. thus such a censoring consists of $m$, and $\left(g_{1}, g_{2}, \cdots, g_{m}\right)$, such that $g_{1}+g_{2}+\cdots+g_{m}=M-m$. This scheme contains Type-II censoring as a particular case (when $g_{1}=\cdots=g_{m-1}=0$ and $g_{m}=M-m$ ) and complete sample scheme (when $M=m$ and $\left.g_{1}=\cdots=g_{m}=0\right)$ is also a special case. This method has attracted attention of many researchers, see, [3], [4],[5], [10], [11], [24], [27], [33].

In many studies, better inference procedures is required for unknown parametric functions such as inference problems involving estimation of multicomponent system reliability which has received great attention in literature recently. Multicomponent system is a system having more than one component. Studies on system reliability was first carried out by [8]. Deriving useful inferences for multicomponent system reliability is of practical interest in many applications such as analysis and design of bridge structures, communication engineering, building defence systems, reliability engineering, radio communications, industrial experiments etc. Such a system with $k_{0}$ strength (independent) variables like $X_{1}, X_{2}, \ldots, X_{k}$ performs adequately if at least $s-o u t-o f-k$ of such variables overcome random stress $T$ experienced by the structures. This is known as $(s-o u t-o f-k: G)$ system where $s \leq k$. Based on complete sample, several researchers have derived inferences for multicomponent system reliability. Some recent studies in this field are: [2], [12], [13], [17], [19], [21], [20]. Despite plenty of work done on multicomponent system reliability based on complete sample, however, inferences on multicomponent reliability based on censoring are scanty. The very few that we are aware of are: [22] evaluated different estimators of multicomponent reliability based on progressive censored data assuming Kumarswamy distribution. The author mentioned that such inferences can be applied in some other context of reliability applications. Different other censoring methods can also be applied to obtain similar inferences. Using progressively censored observations, [26] derived inference for reliability when the stress-strength follow Burr XII distribution. Various useful estimates of parametric function are discussed. Their behavior is assessed via simulations. Recently, [17] obtained different estimates for the multicomponent system reliability assuming unit Gompertz distribution. Such investigation can also be extended to the situation where stress and strength variables are not taken from same probability distribution. One may also refer to [1] and [18] for some further literature on such problems.

Motivation of this work is three-fold: First objective is to obtain MLEs, the asymptotic confidence interval and two bootstarp confidence intervals of $R_{s, k}$ when both the shape parameters are unknown and one of the shape parameter is known. Second objective is to obtain the Bayes estimates of $R_{s, k}$ when both the shape parameters are unknown and one of the shape parameter is known by using Lindley's approximation and the MCMC methods under quadratic loss function using independent gamma priors. In addition, the highest posterior density (HPD) credible interval for $R_{s, k}$ is constructed. Third objective is to obtain uniformly minimum variance unbiased estimator (UMVUE) of system reliability $R_{s, k}$ when common shape parameter is known. As far as we know, no study has attempted to study multicomponent system reliability under progressively type II censored data based on unit Weibull distribution using above methods of estimation.

In Section 2, estimation of proposed parametric function is considered when parameters $\left(\alpha_{1}, \alpha_{2}\right.$ and $\left.\beta\right)$ are unknown. Particularly, maximum likelihood estimator (MLE), approximate confidence and bootstrap intervals are obtained. The Bayes estimators are evaluated against quadratic loss function when priors of unknown parameters follow gamma distributions. In the sequel, the HPD interval is evaluated from MH samples. In Section 3, MLE and approximate interval are obtained under known common parameter. We further evaluate the UMVUE of system reliability. Section 4 deals with simulation study where performance of proposed procedures is evaluated and findings are illustrated in tabular forms. Jute fibre strength data has been analyzed for illustrative purposes in Section 5 . In Section 6, concluding remarks are given. 


\section{Reliability estimation under un- known common parameter}

Here we obtain various point estimates of the unknown parametric function. Interval estimation is also discussed. Let $\left(X_{i 1}, X_{i 2}, \ldots, X_{i k}\right), \quad i=1, \ldots, m$, be the censored data taken from $\operatorname{UW}\left(\alpha_{1}, \beta\right)$ distribution using the scheme $\left(K, k, g_{1}, g_{2}, \ldots, g_{k}\right)$. Further assume that $\left(T_{1}, T_{2} \ldots, T_{m}\right)$ are a likewise data from the $\mathrm{UW}\left(\alpha_{2}, \beta\right)$ distribution obtained from the scheme $\left(M, m, f_{1}, f_{2}, \ldots, f_{m}\right)$. The associated system reliability of the model is then obtained as

$$
\begin{aligned}
R_{s, k}= & \sum_{i=s}^{k}\left(\begin{array}{l}
k \\
i
\end{array}\right) \int_{-\infty}^{\infty}\left[1-F_{X}(t)\right]^{i}\left[F_{X}(t)\right]^{k-i} d F_{T}(t) \\
& =\sum_{i=s}^{k} \sum_{j=0}^{i}\left(\begin{array}{l}
i \\
j
\end{array}\right)\left(\begin{array}{l}
k \\
i
\end{array}\right)(-1)^{j} \alpha_{2}\left[\alpha_{1}(j+k-i)+\alpha_{2}\right]^{-1} .(3)
\end{aligned}
$$

\subsection{Maximum likelihood estimation}

In order to obtain MLE of the system reliability, we first evaluate MLEs of $\alpha_{1}, \alpha_{2}$ and $\beta$ based on progressive type II censored samples. Suppose that a total of $M$ systems are subjected to a test. Each of these consists of $K$ components. Subsequently failure times of $m$ systems each consisting $k$ components are recorded. Then stress-strength samples are obtained as follows:

$$
\left(\begin{array}{cccc}
X_{11} & X_{12} & \ldots & X_{1 k} \\
\vdots & \vdots & \ddots & \vdots \\
X_{m 1} & X_{m 2} & \ldots & X_{m k}
\end{array}\right) \text { and }\left[\begin{array}{c}
T_{1} \\
\vdots \\
T_{m}
\end{array}\right] .
$$

The likelihood function of $\alpha_{1}, \alpha_{2}$ and $\beta$ is

$L\left(\alpha_{1}, \alpha_{2}, \beta\right.$; data $) \propto \prod_{i=1}^{m}\left(\prod_{j=1}^{k} f\left(x_{i j}\right)\left[1-F\left(x_{i j}\right)\right]^{g_{j}}\right) f\left(t_{i}\right)\left[1-F\left(t_{i}\right)\right]^{f_{i}}$. For considered stress-strength model, we have

$L\left(\alpha_{1}, \alpha_{2}, \beta\right.$; data $) \propto \prod_{i=1}^{m}\left(\prod_{j=1}^{k} \alpha_{1} \beta\left(-\ln x_{i j}\right)^{\beta-1} x_{i j}^{-1} e^{-\alpha_{1}\left(-\ln x_{i j}\right)^{\beta}}\left(1-e^{-\alpha_{1}\left(-\ln x_{i j}\right)^{\beta}}\right)^{g_{j}}\right)$

$$
\alpha_{2} \beta\left(-\ln t_{i}\right)^{\beta-1} t_{i}^{-1} e^{-\alpha_{2}\left(-\ln t_{i}\right)^{\beta}}\left(1-e^{-\alpha_{2}\left(-\ln y_{i}\right)^{\beta}}\right)^{f_{i}} .
$$

The log-likelihood is given by

$$
\begin{aligned}
l\left(\alpha_{1}, \alpha_{2}, \beta, \text { data }\right)= & (m k) \log \alpha_{1}+m\left(\log \alpha_{2}\right)+(k+1) m \log \beta \\
& +\sum_{i=1}^{m} \sum_{j=1}^{k} \log \left(x_{i j}^{-1}\right) \\
& +(\beta-1) \sum_{i=1}^{m} \sum_{j=1}^{k} \log \left(-\log x_{i j}\right) \\
& +\sum_{i=1}^{m} \sum_{j=1}^{k} g_{j} \log \left(1-e^{-\alpha_{1}\left(-\ln x_{i j}\right)^{\beta}}\right) \\
& +\sum_{i=1}^{m} \log \left(t_{i}^{-1}\right)+(\beta-1) \sum_{i=1}^{m} \log \left(-\log t_{i}\right) \\
& +\sum_{i=1}^{m} f_{i} \log \left(1-e^{-\alpha_{2}\left(-\ln t_{i}\right)^{\beta}}\right) \\
& -\alpha_{1} \sum_{i=1}^{m} \sum_{j=1}^{k}\left(-\log x_{i j}\right)^{\beta}-\alpha_{2} \sum_{i=1}^{m}\left(-\log t_{i}\right)^{\beta}
\end{aligned}
$$

The likelihood equations of parameters are obtained as

$$
\begin{aligned}
& \frac{\partial l}{\partial \alpha_{1}}=\frac{m k}{\alpha_{1}}+\sum_{i=1}^{m} \sum_{j=1}^{k} g_{j} \frac{e^{-\alpha_{1}\left(-\log x_{i j}\right)^{\beta}}\left(-\ln x_{i j}\right)^{\beta}}{\left(1-e^{-\alpha_{1}\left(-\log x_{i j}\right)^{\beta}}\right)} \\
& -\sum_{i=1}^{m} \sum_{j=1}^{k}\left(-\log x_{i j}\right)^{\beta}=0, \\
& \frac{\partial l}{\partial \alpha_{2}}=\frac{m}{\alpha_{2}}+\sum_{i=1}^{m} f_{i} \frac{e^{-\alpha_{2}\left(-\ln t_{i}\right)^{\beta}}\left(-\ln t_{i}\right)^{\beta}}{\left(1-e^{-\alpha_{2}\left(-\ln t_{i}\right)^{\beta}}\right)}-\sum_{i=1}^{m}\left(-\log t_{i}\right)^{\beta}=0, \\
& \text { and } \\
& \begin{aligned}
\frac{\partial l}{\partial \beta}= & \frac{(m k+m)}{\beta}+\sum_{i=1}^{m} \sum_{j=1}^{k} \log \left(-\log x_{i j}\right)+\sum_{i=1}^{m} \log \left(-\log t_{i}\right) \\
& +\sum_{i=1}^{m} \sum_{j=1}^{k} \alpha_{1} g_{j} \frac{e^{-\alpha_{1}\left(-\ln x_{i j}\right)^{\beta}}\left(-\ln x_{i j}\right)^{\beta}}{\left(1-e^{-\alpha_{1}\left(-\ln x_{i j}\right)^{\beta}}\right)} \log \left(-\log x_{i j}\right) \\
& +\sum_{i=1}^{m} \alpha_{2} f_{i} \frac{e^{-\alpha_{2}\left(-\ln t_{i}\right)^{\beta}}\left(-\ln t_{i}\right)^{\beta}}{\left(1-e^{-\alpha_{2}\left(-\ln t_{i}\right)^{\beta}}\right)} \log \left(-\log t_{i}\right) \\
& -\alpha_{1} \sum_{i=1}^{m} \sum_{j=1}^{k} \log \left(-\log x_{i j}\right)\left(-\log x_{i j}\right)^{\beta} \\
& -\alpha_{2} \sum_{i=1}^{m} \log \left(-\log t_{i}\right)\left(-\log t_{i}\right)^{\beta}=0
\end{aligned}
\end{aligned}
$$
numerical method to evaluate respective $\operatorname{MLEs}\left(\hat{\alpha_{1}}, \hat{\alpha_{2}}, \hat{\beta}\right)$ of parameters $\left(\alpha_{1}, \alpha_{2}, \beta\right)$. The maximum likelihood estimator of system reliability is then obtained as

$$
R_{s, k}^{M}=\sum_{i=s}^{k} \sum_{j=0}^{i}\left(\begin{array}{l}
i \\
j
\end{array}\right)\left(\begin{array}{l}
k \\
i
\end{array}\right)(-1)^{j} \hat{\alpha}_{2}\left[\hat{\alpha}_{1}(j+k-i)+\hat{\alpha}_{2}\right]^{-1} \text {. }
$$

\section{$2.2 \quad$ Asymptotic interval}

In this subsection, asymptotic interval of unknown parametric function is derived using the censored data. The expected Fisher information matrix is given by $J(\theta)=-E(I(\theta))$, where $I(\theta)=\left[I_{i j}\right]=\left[-\frac{\partial^{2} l}{\partial \theta_{i} \partial \theta_{j}}\right], \quad i, j=1,2,3$. The elements of this matrix are evaluated as follows:

$$
\begin{aligned}
I_{11}= & -\frac{m k}{\alpha_{1}^{2}}-\sum_{i=1}^{m} \sum_{j=1}^{k} g_{j}\left(-\log x_{i j}\right)^{2 \beta} \frac{e^{-\alpha_{1}\left(-\log x_{i j}\right)^{\beta}}}{\left(1-e^{-\alpha_{1}\left(-\log x_{i j}\right)^{\beta}}\right)^{2}} \\
I_{22}= & -\frac{m}{\alpha_{2}^{2}}-\sum_{i=1}^{m} f_{i}\left(-\log t_{i}\right)^{2 \beta} \frac{e^{-\alpha_{2}\left(-\log t_{i}\right)^{\beta}}}{\left(1-e^{\left.-\alpha_{2}\left(-\log t_{i}\right)^{\beta}\right)^{2}}\right.} \\
& I_{21}=0, \quad I_{12}=0, \\
I_{13}= & \sum_{i=1}^{m} \sum_{j=1}^{k} g_{j} e^{-\alpha_{1}\left(-\log x_{i j}\right)^{\beta}}\left(-\log x_{i j}\right)^{\beta} \times \\
& \left(-\log x_{i j}\right) \frac{\left(1-\alpha_{1}\left(-\log x_{i j}\right)^{\beta}\right)}{\left(1-e^{-\alpha_{1}\left(-\log x_{i j}\right)^{\beta}}\right)} \times \\
& -\sum_{i=1}^{m} \sum_{j=1}^{k} \log \left(-\log x_{i j}\right)\left(-\log x_{i j}\right)^{\beta}
\end{aligned}
$$




$$
\begin{aligned}
I_{23}= & \sum_{i=1}^{m} f_{i} e^{-\alpha_{2}\left(-\log t_{i}\right)^{\beta}}\left(-\log t_{i}\right)^{\beta}\left(-\log t_{i}\right) \frac{\left(1-\alpha_{2}\left(-\log t_{i}\right)^{\beta}\right)}{\left(1-e^{-\alpha_{2}\left(-\log t_{i}\right)^{\beta}}\right)} \\
& -\sum_{i=1}^{m} \log \left(-\log t_{i}\right)\left(-\log t_{i}\right)^{\beta} \\
I_{33}= & -\frac{m(1+k)}{\beta^{2}}+\sum_{i=1}^{m} \sum_{j=1}^{k} g_{j} \alpha_{1}\left(\log \left(-\log x_{i j}\right)\right) e^{-\alpha_{1}\left(-\log x_{i j}\right)^{\beta}} \\
& \left(-\log x_{i j}\right)^{\beta}\left(-\log x_{i j}\right) \frac{\left(1-\alpha_{1}\left(-\log x_{i j}\right)^{\beta}\right)}{\left(1-e^{\left.-\alpha_{1}\left(-\log x_{i j}\right)^{\beta}\right)}\right.} \\
& +\sum_{i=1}^{m} f_{i} \alpha_{2}\left(\log \left(-\log t_{i}\right)\right) e^{-\alpha_{2}\left(-\log t_{i}\right)^{\beta}}\left(-\log t_{i}\right)^{\beta}\left(-\log t_{i}\right) \\
& \frac{\left(1-\alpha_{2}\left(-\log t_{i}\right)^{\beta}\right)}{\left(1-e^{\left.-\alpha_{2}\left(-\log t_{i}\right)^{\beta}\right)}-\alpha_{1} \sum_{i=1}^{m} \sum_{j=1}^{k}\left(\log \left(-\log x_{i j}\right)\right)^{2}\left(-\log x_{i j}\right)^{\beta}\right.} \\
& -\alpha_{2} \sum_{i=1}^{m}\left(\log \left(-\log t_{i}\right)\right)^{2}\left(-\log t_{i}\right)^{\beta}
\end{aligned}
$$

The MLE $\hat{R}_{s, k}$ is normally distributed having average value $R_{s, k}$ and variance

$$
\sigma_{R_{s, k}}^{2}=\left(\frac{\partial R_{s, k}}{\partial \alpha_{1}}\right)^{2} J_{11}^{-1}+\left(\frac{\partial R_{s, k}}{\partial \alpha_{2}}\right)^{2} J_{22}^{-1}+2\left(\frac{\partial R_{s, k}}{\partial \alpha_{1}}\right)\left(\frac{\partial R_{s, k}}{\partial \alpha_{2}}\right) J_{12}^{-1},
$$

where

$$
\begin{aligned}
\frac{\partial R_{s, k}}{\partial \alpha_{1}}= & \sum_{i=s}^{k} \sum_{j=0}^{i}\left(\begin{array}{l}
k \\
i
\end{array}\right)\left(\begin{array}{l}
i \\
j
\end{array}\right)(-1)^{j+1} \alpha_{2}(j+k-i) \times \\
& {\left[\alpha_{1}(j+k-i)+\alpha_{2}\right]^{-2} } \\
\frac{\partial R_{s, k}}{\partial \alpha_{2}}= & \sum_{i=s}^{k} \sum_{j=0}^{i}\left(\begin{array}{l}
k \\
i
\end{array}\right)\left(\begin{array}{l}
i \\
j
\end{array}\right)(-1)^{j}(k-i+j) \alpha_{1} \times \\
& {\left[\alpha_{1}(k-i+j)+\alpha_{2}\right]^{-2} . }
\end{aligned}
$$

Now a $100(1-\eta) \%, 0<\eta<1$, interval of the reliability is computed as $\left(\hat{R}_{s, k} \pm q_{\eta / 2} \hat{\sigma}_{R_{s, k}}\right)$, where $q_{\eta / 2}$ denotes the uper $(\eta / 2)$ th of $N(0,1)$.

\section{$2.3 \quad$ Bootstrap intervals}

Now boot-p and boot-t intervals of the reliability are evaluated based under progressive type II censoring. Such intervals are highly popular in statistical literature. Readers may refer to [14] and [15] for detailed discussion on these methods.

\subsubsection{Boot-P Method}

1. Simulate data $\left(t_{1}^{*}, \ldots, t_{m}^{*}\right)$ of size $m$. Further generate data $\left(x_{i 1}^{*}, \ldots, x_{i k}^{*}\right), i=1,2, \ldots, m$, of size $n k$. Bootstrap estimate of $R_{s, k}$ is evaluated as $\hat{R}_{s, k}^{*}$.

2. Repeat this step sufficient number of times.

3. Let $F^{*}(x)$ be the empirical cdf of bootstrap estimate $\hat{R}_{s, k}^{*}$. Consider $\hat{R}_{s, k}^{b p}(x)=F^{*-1}(x)$, then $100(1-\eta) \%$ boot-p interval turns out to be $\left(\hat{R}_{s, k}^{b p}\left(\frac{\eta}{2}\right), \hat{R}_{s, k}^{b p}\left(1-\frac{\eta}{2}\right)\right)$.

\subsubsection{Boot-T Method}

1. Generate data $\left(t_{1}^{*}, \ldots, t_{m}^{*}\right)$ of size $m$. Further generate data $\left(x_{i 1}^{*}, \ldots, x_{i k}^{*}\right) i=1,2, \ldots, m$, of size $m k$. Bootstrap estimate of $R_{s, k}$ is evaluated as $\hat{R}_{s, k}^{*}$. Compute $T^{*}=$ $\left(\sqrt{V\left(\hat{R}_{s, k}^{*}\right)}\right)^{-1}\left(\hat{R}_{s, k}^{*}-\hat{R}_{s, k}\right)$

2. Repeat these two steps sufficient number of times.

3. Let $H_{T^{*}}(x)$ be the empirical cdf of $T^{*}$ and also $\hat{R}_{s, k}^{b t}(x)=$ $\hat{R}_{s, k}(x)+H_{T^{*}}^{-1}(x) \sqrt{V\left(\hat{R}_{s, k}\right)}$. The required boot-t interval turns out to be $\left(\hat{R}_{s, k}^{b t}\left(\frac{\eta}{2}\right), \hat{R}_{s, k}^{b t}\left(1-\frac{\eta}{2}\right)\right)$.

\subsection{Bayesian inference}

In this subsection, Bayesian approach is applied for estimating the reliability. Bayesian analysis is a natural way to combine the observed information with the prior distribution which makes it reasonably useful in reliability, lifetime study, survival analysis. To simplify the Bayesian analysis, we consider independent gamma priors for $\alpha_{1}, \alpha_{2}$ and $\beta$ with parameters $\left(p_{i}, q_{i}\right)$, where $p_{i}$ and $q_{i}$ are the shape and scale parameters, $i=1,2,3$. Thus the prior distribution is of the form

$$
f^{i}(x)=\frac{q_{i}^{p_{i}}}{\Gamma\left(p_{i}\right)} x^{p_{i}-1} e^{-x q_{i}}, x>0, p_{i}>0, q_{i}>0, i=1,2,3 .
$$

Subsequently joint posterior is of the following form:

$$
\begin{aligned}
& \pi\left(\alpha_{1}, \alpha_{2}, \beta \mid \text { data }\right) \propto \alpha_{1}^{m k+p_{1}-1} \alpha_{2}^{m+p_{2}-1} \beta^{m(k+1)+p_{3}-1} \\
& e^{-\alpha_{1}\left(q_{1}+\sum_{i=1}^{m} \sum_{j=1}^{k}\left(-\log x_{i j}\right)^{\beta}\right)} e^{-\alpha_{2}\left(q_{2}+\sum_{i=1}^{m}\left(-\log t_{i}\right)^{\beta}\right)} \\
& e^{\sum_{i=1}^{m} \sum_{j=1}^{k} g_{j} \log \left(1-e^{-\alpha_{1}\left(-\log x_{i j}\right)^{\beta}}\right)} e^{\sum_{i=1}^{m} f_{i} \log \left(1-e^{-\alpha_{2}\left(-\log t_{i}\right)^{\beta}}\right)} \\
& e^{-\beta\left(q_{3}-\sum_{i=1}^{m} \sum_{j=1}^{k}\left(-\log x_{i j}\right)-\sum_{i=1}^{m}\left(-\log t_{i}\right)\right)} .
\end{aligned}
$$

Note that normalizing constant is not helpful in evaluating the Bayes estimate. The estimate of $R_{s, k}$ is computed under the quadratic loss function where desired estimate is the posterior mean of $R_{s, k}$. Subsequently the desired estimator from above posterior is obtained as

$$
\tilde{R}_{s, k}^{B}=\int_{0}^{\infty} \int_{0}^{\infty} \int_{0}^{\infty} R_{s, k} \pi\left(\alpha_{1}, \alpha_{2}, \beta \mid \text { data }\right) d \alpha_{1} d \alpha_{2} d \beta .
$$

This estimator is the ratio of two integrals. We are not able to solve it explicitly as the posterior distribution is intractable form, thus we use Lindley's approximation method (see, [25]) and $\mathrm{MH}$ algorithm (see, [32], [16]) to compute the estimate $\tilde{R}_{s, k}^{B}$ of $R_{s, k}$.

\subsubsection{Lindley's approximation method}

Now the required estimate of $R_{s, k}$ is evaluated using the Lindley's method. Consider the posterior expectation of the function $u(\theta)$ such as $E(u(\theta) \mid x, y)=\int u(\theta) e^{l(\theta)+\rho(\theta)} d \theta / \int e^{l(\theta)+\rho(\theta)} d \theta$, where $l(\theta)$ and $\rho(\theta)$ are log-likelihood function of $\theta$ and logarithm of prior distribution, respectively and $\theta=\left(\alpha_{1}, \alpha_{2}, \beta\right)$. The 
Bayes estimate turns out to be as follows:

$$
\begin{aligned}
\tilde{R}_{s, k}= & u(\theta)+\left(u_{1} a_{1}+u_{2} a_{2}+u_{3} a_{3}+a_{4}+a_{5}\right)+ \\
& 0.5\left[\left(\sigma_{11} l_{111}+2 \sigma_{12} l_{121}+2 \sigma_{13} l_{131}+2 \sigma_{23} l_{231}+\right.\right. \\
& \left.\sigma_{22} l_{221}+\sigma_{33} l_{331}\right)\left(u_{1} \sigma_{11}+u_{2} \sigma_{12}+u_{3} \sigma_{13}\right)+\left(\sigma_{11} l_{112}\right. \\
& +2 \sigma_{12} l_{122}+2 \sigma_{13} l_{132}+2 \sigma_{23} l_{232}+ \\
& \left.\sigma_{22} l_{222}+\sigma_{33} l_{332}\right)\left(u_{1} \sigma_{21}+u_{2} \sigma_{22}+u_{3} \sigma_{23}\right) \\
& +\left(\sigma_{11} l_{113}+2 \sigma_{12} l_{123}+2 \sigma_{13} l_{133}+2 \sigma_{23} l_{233}+\right. \\
& \left.\left.\sigma_{22} l_{223}+\sigma_{33} l_{333}\right)\left(u_{1} \sigma_{31}+u_{2} \sigma_{32}+u_{3} \sigma_{33}\right)\right] .
\end{aligned}
$$

Respective quantities are defined as $a_{i}=\rho_{1} \sigma_{i 1}+\rho_{2} \sigma_{i 2}+$ $\rho_{3} \sigma_{i 3}, \quad i=1,2,3, \quad a_{4}=u_{12} \sigma_{12}+u_{13} \sigma_{13}+u_{23} \sigma_{23}, \quad a_{5}=$ $0.5\left(u_{11} \sigma_{11}+u_{22} \sigma_{22}+u_{33} \sigma_{33}\right), \rho_{1}=\frac{p_{1}-1}{\alpha_{1}}-q_{1}, \rho_{2}=\frac{p_{2}-1}{\alpha_{2}}-$ $q_{2}, \rho_{3}=\frac{p_{3}-1}{\beta}-q_{3}, \sigma_{i k}$ is given as $\left[-l_{i k}\right]^{-1}, i, k=1,2,3$. We further take $u(\theta)$ as $R_{s, k}$. All quantities evaluated at maximum likelihood estimates. Other quantities of Eqn. (10) are presented below:

$$
\begin{aligned}
l_{1}=\frac{m k}{\alpha_{1}}+ & \sum_{i=1}^{m} \sum_{j=1}^{k} g_{j} \frac{e^{-\alpha_{1}\left(-\log x_{i j}\right)^{\beta}}\left(-\ln x_{i j}\right)^{\beta}}{\left(1-e^{-\alpha_{1}\left(-\log x_{i j}\right)^{\beta}}\right)}-\sum_{i=1}^{m} \sum_{j=1}^{k}\left(-\log x_{i j}\right)^{\beta} \\
l_{2}= & \frac{m}{\alpha_{2}}+\sum_{i=1}^{m} f_{i} \frac{e^{-\alpha_{2}\left(-\ln t_{i}\right)^{\beta}}\left(-\ln t_{i}\right)^{\beta}}{\left(1-e^{-\alpha_{2}\left(-\ln t_{i}\right)^{\beta}}\right)}-\sum_{i=1}^{m}\left(-\log t_{i}\right)^{\beta} \\
l_{3}= & \frac{(m k+m)}{\beta}+\sum_{i=1}^{m} \sum_{j=1}^{k} \log \left(-\log x_{i j}\right)+\sum_{i=1}^{m} \log \left(-\log t_{i}\right) \\
& +\sum_{i=1}^{m} \sum_{j=1}^{k} \alpha_{1} g_{j} \frac{e^{-\alpha_{1}\left(-\ln x_{i j}\right)^{\beta}}\left(-\ln x_{i j}\right)^{\beta}}{\left(1-e^{-\alpha_{1}\left(-\ln x_{i j}\right)^{\beta}}\right)} \log \left(-\log x_{i j}\right) \\
& +\sum_{i=1}^{m} \alpha_{2} f_{i} \frac{e^{-\alpha_{2}\left(-\ln t_{i}\right)^{\beta}}\left(-\ln t_{i}\right)^{\beta}}{\left(1-e^{-\alpha_{2}\left(-\ln t_{i}\right)^{\beta}}\right)} \log \left(-\log t_{i}\right) \\
& -\alpha_{1} \sum_{i=1}^{m} \sum_{j=1}^{k}\left(\log \left(-\log x_{i j}\right)\right)^{2}\left(-\log x_{i j}\right)^{\beta} \\
& -\alpha_{2} \sum_{i=1}^{m}\left(\log \left(-\log t_{i}\right)\right)^{2}\left(-\log t_{i}\right)^{\beta} .
\end{aligned}
$$

Also $l_{11}, l_{22}, l_{33}, l_{13}, l_{23}$ are evaluated in Section 2.2. Note also $l_{12}=0$ and $l_{21}=0$. Further

$$
\begin{aligned}
& l_{111}=\frac{2 m k}{\alpha_{1}^{3}}+\sum_{i=1}^{m} \sum_{j=1}^{k} g_{j}\left[1+e^{-\alpha_{1}\left(-\log x_{i j}\right)^{\beta}}\right] \\
& \frac{e^{-\alpha_{1}\left(-\log x_{i j}\right)^{\beta}}\left(-\ln x_{i j}\right)^{3 \beta}}{\left(1-e^{-\alpha_{1}\left(-\log x_{i j}\right)^{\beta}}\right)^{3}} \\
& l_{222}=\frac{2 m}{\alpha_{2}^{3}}+\sum_{i=1}^{m} f_{i}\left[1+e^{-\alpha_{2}\left(-\log t_{i}\right)^{\beta}}\right] \frac{e^{-\alpha_{2}\left(-\log t_{i}\right)^{\beta}}\left(-\ln t_{i}\right)^{3 \beta}}{\left(1-e^{-\alpha_{2}\left(-\log t_{i}\right)^{\beta}}\right)^{3}} \\
& -l_{113}=\sum_{i=1}^{m} \sum_{j=1}^{k} g_{j}\left(-\ln x_{i j}\right)^{2 \beta} \log \left(-\ln x_{i j}\right) e^{-\alpha_{1}\left(-\log x_{i j}\right)^{\beta}} \\
& \left(1-e^{-\alpha_{2}\left(-\log x_{i j}\right)^{\beta}}\right)^{-3} \\
& \left\{\left[2-\alpha_{1}\left(-\ln x_{i j}\right)^{\beta}\right]\left[1-e^{-\alpha_{1}\left(-\log x_{i j}\right)^{\beta}}\right]\right. \\
& \left.-2 \alpha_{1} e^{-\alpha_{1}\left(-\log x_{i j}\right)^{\beta}}\left(-\ln x_{i j}\right)^{\beta}\right\}
\end{aligned}
$$$$
-l_{223}=\sum_{i=1}^{m} f_{i}\left(-\ln t_{i}\right)^{2 \beta} \log \left(-\ln t_{i}\right) e^{-\alpha_{2}\left(-\log t_{i}\right)^{\beta}}
$$$$
\left(1-e^{-\alpha_{2}\left(-\log t_{i}\right)^{\beta}}\right)^{-3}
$$$$
\left\{\left[2-\alpha_{2}\left(-\ln t_{i}\right)^{\beta}\right]\left[1-e^{-\alpha_{2}\left(-\log t_{i}\right)^{\beta}}\right]\right.
$$$$
\left.-2 \alpha_{2} e^{-\alpha_{2}\left(-\log t_{i}\right)^{\beta}}\left(-\ln t_{i}\right)^{\beta}\right\}
$$$$
l_{333}=\sum_{i=1}^{m} \sum_{j=1}^{k} \alpha_{1} g_{j}\left(-\ln x_{i j}\right)^{\beta}\left(-\ln x_{i j}\right)\left(\log \left(-\ln x_{i j}\right)\right)^{2}
$$$$
e^{-\alpha_{1}\left(-\log x_{i j}\right)^{\beta}}\left(1-e^{-\alpha_{1}\left(-\log x_{i j}\right)^{\beta}}\right)^{-2}
$$$$
\left\{\left[1-2 \alpha_{1}\left(-\ln x_{i j}\right)^{\beta}-\alpha_{1}\left(-\ln x_{i j}\right)^{\beta}\left(1-\alpha_{1}\left(-\ln x_{i j}\right)^{\beta}\right)\right]\right.
$$$$
\left[1-e^{-\alpha_{1}\left(-\log x_{i j}\right)^{\beta}}\right]
$$$$
\left.-\alpha_{1}\left(-\ln x_{i j}\right)^{\beta} e^{-\alpha_{1}\left(-\log x_{i j}\right)^{\beta}}\left(1-\alpha_{1}\left(-\ln x_{i j}\right)^{\beta}\right)\right\}
$$$$
+\frac{2 m(k+1)}{\beta^{3}}+\sum_{i=1}^{m} \alpha_{2} f_{i}\left(-\ln t_{i}\right)^{\beta}\left(-\ln t_{i}\right)
$$$$
\left(\log \left(-\ln t_{i}\right)\right)^{2} e^{-\alpha_{2}\left(-\log t_{i}\right)^{\beta}}\left(1-e^{-\alpha_{2}\left(-\log t_{i}\right)^{\beta}}\right)^{-2}
$$$$
\left\{\left[1-2 \alpha_{2}\left(-\ln t_{i}\right)^{\beta}-\alpha_{2}\left(-\ln t_{i}\right)^{\beta}\right.\right.
$$$$
\left.\left(1-\alpha_{2}\left(-\ln t_{i}\right)^{\beta}\right)\right]\left[1-e^{-\alpha_{2}\left(-\log t_{i}\right)^{\beta}}\right]
$$$$
\left.-\alpha_{2}\left(-\ln t_{i}\right)^{\beta} e^{-\alpha_{2}\left(-\log t_{i}\right)^{\beta}}\left(1-\alpha_{2}\left(-\ln t_{i}\right)^{\beta}\right)\right\}
$$$$
-\alpha_{1} \sum_{i=1}^{m} \sum_{j=1}^{k}\left(\log \left(-\log x_{i j}\right)\right)^{3}\left(-\log x_{i j}\right)^{\beta}
$$$$
-\alpha_{2} \sum_{i=1}^{m}\left(\log \left(-\log t_{i}\right)\right)^{3}\left(-\log t_{i}\right)^{\beta}
$$$$
l_{331}=\sum_{i=1}^{m} \sum_{j=1}^{k} g_{j}\left(-\ln x_{i j}\right)^{\beta}\left(-\ln x_{i j}\right)\left(\log \left(-\ln x_{i j}\right)\right)
$$$$
e^{-\alpha_{1}\left(-\log x_{i j}\right)^{\beta}}\left(1-e^{-\alpha_{1}\left(-\log x_{i j}\right)^{\beta}}\right)^{-1}
$$$$
\left\{\left[1-2 \alpha_{1}\left(-\ln x_{i j}\right)^{\beta}-\alpha_{1}\left(-\ln x_{i j}\right)^{\beta}\left(1-\alpha_{1}\left(-\ln x_{i j}\right)^{\beta}\right)\right]\right.
$$$$
\left.\left[1-e^{-\alpha_{1}\left(-\log x_{i j}\right)^{\beta}}\right]^{-1}\right\}
$$$$
-\sum_{i=1}^{m} \sum_{j=1}^{k} \log \left(-\log x_{i j}\right)^{2}\left(-\log x_{i j}\right)^{\beta}
$$$$
l_{332}=\sum_{i=1}^{m} f_{i}\left(-\ln t_{i}\right)^{\beta}\left(-\ln t_{i}\right)\left(\log \left(-\ln t_{i}\right)\right) e^{-\alpha_{2}\left(-\log t_{i}\right)^{\beta}}
$$$$
\left(1-e^{-\alpha_{2}\left(-\log t_{i}\right)^{\beta}}\right)^{-1}
$$$$
\left\{\left[1-2 \alpha_{2}\left(-\ln t_{i}\right)^{\beta}-\alpha_{2}\left(-\ln t_{i}\right)^{\beta}\left(1-\alpha_{2}\left(-\ln t_{i}\right)^{\beta}\right)\right]\right.
$$$$
\left.\left[1-e^{-\alpha_{2}\left(-\log t_{i}\right)^{\beta}}\right]^{-1}\right\}
$$$$
-\sum_{i=1}^{m} \log \left(-\log t_{i}\right)^{2}\left(-\log t_{i}\right)^{\beta}
$$$$
l_{132}=l_{122}=l_{123}=l_{231}=l_{221}=l_{112}=l_{121}=0,
$$

Considering $u(\theta)=R_{S, k}$, we have

$$
u_{1}=\frac{\partial R_{s, k}}{\partial \alpha_{1}}=\sum_{i=s}^{k} \sum_{j=0}^{i}\left(\begin{array}{l}
k \\
i
\end{array}\right)\left(\begin{array}{l}
i \\
j
\end{array}\right)(-1)^{j+1} \frac{\alpha_{2}(j+k-i)}{\left[\alpha_{1}(j+k-i)+\alpha_{2}\right]^{2}},
$$




$$
\begin{aligned}
& u_{2}=\frac{\partial R_{s, k}}{\partial \alpha_{2}}=\sum_{i=s}^{k} \sum_{j=0}^{i}\left(\begin{array}{l}
k \\
i
\end{array}\right)\left(\begin{array}{l}
i \\
j
\end{array}\right)(-1)^{j} \frac{\alpha_{1}(j+k-i)}{\left[\alpha_{1}(j+k-i)+\alpha_{2}\right]^{2}} \\
& u_{11}=\frac{\partial^{2} R_{s, k}}{\partial \alpha_{1}^{2}}=\sum_{i=s}^{k} \sum_{j=0}^{i}\left(\begin{array}{l}
k \\
i
\end{array}\right)\left(\begin{array}{l}
i \\
j
\end{array}\right)(-1)^{j} \frac{2 \alpha_{2}(j+k-i)^{2}}{\left[\alpha_{1}(j+k-i)+\alpha_{2}\right]^{3}}, \\
& u_{22}=\frac{\partial^{2} R_{s, k}}{\partial \alpha_{2}^{2}}=\sum_{i=s}^{k} \sum_{j=0}^{i}\left(\begin{array}{l}
k \\
i
\end{array}\right)\left(\begin{array}{l}
i \\
j
\end{array}\right)(-1)^{j+1} \frac{2 \alpha_{1}(j+k-i)}{\left[\alpha_{1}(j+k-i)+\alpha_{2}\right]^{3}}, \\
& u_{3}=\frac{\partial R_{s, k}}{\partial \beta}=0, \quad u_{33}=u_{13}=u_{23}=0, \\
& u_{12}=u_{21}=\frac{\partial^{2} R_{s, k}}{\partial \alpha_{1} \partial \alpha_{2}}=\sum_{i=s}^{k} \sum_{j=0}^{i}\left(\begin{array}{l}
k \\
i
\end{array}\right)\left(\begin{array}{l}
i \\
j
\end{array}\right) \\
&
\end{aligned}
$$

This Lindley method is very popular for deriving point estimates of various unknown parameters, however, it is not very useful for evaluating credible intervals. We next discuss $\mathrm{MH}$ algorithm which is widely used for computing Bayes point and interval estimates of unknown parametric functions.

\subsubsection{MH algorithm}

Here we apply this method to evaluate Bayes estimate of the system reliability, $R_{s, k}$ and credible intervals under the given censored data. We see that marginal distributions of $\alpha_{1}, \alpha_{2}$ and $\beta$ may not be available in known forms. These marginals are evaluated using normal proposal distributions. The following algorithm is used for computations purposes.

Step 1: Consider initial guess $\left(\alpha_{1_{0}}, \alpha_{2_{0}}, \beta_{0}\right)$ of $\left(\alpha_{1}, \alpha_{2}, \beta\right)$ and set $d=1$.

Step 2: Generate $\beta_{(d)}$ from $N\left(\mu_{d-1}, \sigma^{2}\right)$ at $d$ th step.

Step 3: Generate $\alpha_{1(d)}$ using $N\left(\alpha_{1(d-1)}, \sigma^{2}\right)$.

Step 4: Generate $\alpha_{2(d)}$ using $N\left(\alpha_{2(d-1)}, \sigma^{2}\right)$.

Step 5: Compute $R_{(d)}=\sum_{i=s}^{k} \sum_{j=0}^{i}\left(\begin{array}{l}i \\ j\end{array}\right)\left(\begin{array}{c}k \\ i\end{array}\right)(-1)^{j} \alpha_{2(d)}\left[\alpha_{1(d)}(j+\right.$ $\left.k-i)+\alpha_{2(d)}\right]^{-1}$.

Step 6: Set $d=d+1$.

Step 8: Iterate steps 2-6, $n_{0}$ times.

Bayes estimator of $R_{s, k}$ is now obtained as

$$
\tilde{R}_{s, k}^{M}=\frac{1}{n_{0}} \sum_{i=1}^{n_{0}} R_{(d) s, k} .
$$

The $100(1-\eta) \%$ HPD interval of $R_{s, k}$ is evaluated using the procedure of [9].

\section{Reliability estimation under known common parameter}

Now estimation of $R_{s, k}$ is considered when $\beta$ is known and data are progressive type II censored. We take $\beta=\beta_{0}, \beta_{0}$ is known constant. Different estimators are discussed in this case also.

\subsection{Maximum likelihood estimation}

Here we obtain MLE of unknown parametric function using the observe censored data when $\beta$ is known. The likelihood function turns out to be

$$
\begin{aligned}
& L\left(\alpha_{1}, \alpha_{2}, \text { data }\right) \propto \prod_{i=1}^{m}\left(\prod_{j=1}^{k} \alpha_{1}\left(-\ln x_{i j}\right)^{\beta_{0}-1} x_{i j}^{-1}\right. \\
& \left.\left(1-e^{-\alpha_{1}\left(-\ln x_{i j}\right)^{\beta_{0}}}\right)^{g_{j}} e^{-\alpha_{1}\left(-\ln x_{i j}\right)^{\beta_{0}}}\right) \\
& \alpha_{2}\left(-\ln t_{i}\right)^{\beta_{0}-1} t_{i}^{-1}\left(1-e^{-\alpha_{2}\left(-\ln t_{i}\right)^{\beta_{0}}}\right)^{f_{i}} e^{-\alpha_{2}\left(-\ln t_{i}\right)^{\beta_{0}}} .
\end{aligned}
$$

The log-likelihood function is given by

$$
\begin{aligned}
& l\left(\alpha_{1}, \alpha_{2}, \text { data }\right) \propto(m k) \log \alpha_{1}+m\left(\log \alpha_{2}\right) \\
& +\sum_{i=1}^{m} \sum_{j=1}^{k} g_{j} \log \left(1-e^{-\alpha_{1}\left(-\ln x_{i j}\right)^{\beta}}\right)+\sum_{i=1}^{m} f_{i} \log \left(1-e^{-\alpha_{2}\left(-\ln t_{i}\right)^{\beta}}\right) \\
& -\alpha_{1} \sum_{i=1}^{m} \sum_{j=1}^{k}\left(-\log x_{i j}\right)^{\beta_{0}}-\alpha_{2} \sum_{i=1}^{m}\left(-\log t_{i}\right)^{\beta_{0}}
\end{aligned}
$$

MLEs of $\alpha_{1}$ and $\alpha_{2}$ are solutions of the following equations:

$$
\begin{gathered}
\frac{\partial l}{\partial \alpha_{1}}=\frac{m k}{\alpha_{1}}+\sum_{i=1}^{m} \sum_{j=1}^{k} g_{j} \frac{e^{-\alpha_{1}\left(-\log x_{i j}\right)^{\beta_{0}}}\left(-\ln x_{i j}\right)^{\beta_{0}}}{\left(1-e^{-\alpha_{1}\left(-\log x_{i j}\right)^{\beta_{0}}}\right)} \\
-\sum_{i=1}^{m} \sum_{j=1}^{k}\left(-\log x_{i j}\right)^{\beta_{0}}=0, \\
\frac{\partial l}{\partial \alpha_{2}}=\frac{m}{\alpha_{2}}+\sum_{i=1}^{m} f_{i} \frac{e^{-\alpha_{2}\left(-\ln t_{i}\right)^{\beta_{0}}}\left(-\ln t_{i}\right)^{\beta_{0}}}{\left(1-e^{-\alpha_{2}\left(-\ln t_{i}\right)^{\beta_{0}}}\right)}-\sum_{i=1}^{m}\left(-\log t_{i}\right)^{\beta_{0}}=0,
\end{gathered}
$$

It is seen from the above two non-linear equations that there are no closed form for the MLEs $\hat{\alpha_{1}}$ and $\hat{\alpha_{2}}$. Numerical procedures may be used to solve these nonlinear equations to compute them. The corresponding MLE $\hat{R}_{s, k}$ of system reliability $R_{s, k}$ is computed similarly as earlier. From asymptotic theory, we know that MLE is normally distributed, and accordingly $\hat{R}_{s, k}$ is normal with mean $R_{s, k}$ and variance is

$$
\sigma_{R_{s, k}}^{2}=\left(\frac{\partial R_{s, k}}{\partial \alpha_{1}}\right)^{2} \frac{\alpha_{1}^{2}}{m k}+\left(\frac{\partial R_{s, k}}{\partial \alpha_{2}}\right)^{2} \frac{\alpha_{2}^{2}}{m} .
$$

Hence, a $100(1-\eta) \%$ interval of $R_{s, k}$ is given by $\left(\hat{R}_{s, k} \pm\right.$ $q_{\eta / 2} \hat{\sigma}_{R_{s, k}}$, where $q_{\eta / 2}$ is the upper $(\eta / 2)$ th quantile of $N(0,1)$.

\subsection{UMVUE of $R_{s, k}$}

In this subsection, UMVUE $\hat{R}^{U}$ of multicomponent system reliability is derived. In fact it is enough to evaluate this estimator for $\psi\left(\alpha_{1}, \alpha_{2}\right)=\alpha_{2}\left[\alpha_{1}(j+k-i)+\alpha_{2}\right]^{-1}$.

Let $U^{*}=\sum_{i=1}^{m}\left(f_{i}+1\right)\left(-\log t_{i}\right)^{\beta_{0}}$ and $V^{*}=\sum_{i=1}^{m} \sum_{j=1}^{k}\left(g_{j}+\right.$ $1)\left(-\log x_{i j}\right)^{\beta_{0}}$. Then $\left(U^{*}, V^{*}\right)$ form a complete and sufficient statistic for unknown parameters.

Let $T_{i}^{*}=\left(-\log t_{i}\right)^{\beta_{0}}, i=1,2, \ldots, m$, is a progressively censored sample from one parameter exponential distribution with mean $1 / \alpha_{2}$.

Considering the transformations

$Z_{1}=M T_{1}^{*}$,

$Z_{2}=\left(M-f_{1}-1\right)\left(T_{2}^{*}-T_{1}^{*}\right)$, 
$\cdots$

. .

$Z_{m}=\left(M-f_{1}-\ldots-f_{m-1}-m+1\right)\left(T_{m}^{*}-T_{m-1}^{*}\right)$.

Thus $Z_{1}, Z_{2}, \ldots, Z_{m}$ are iid exponential variables with mean $1 / \alpha_{2}$. So $U^{*}=\sum_{i=1}^{m} Z_{i}=\sum_{i=1}^{m}\left(f_{i}+1\right) T_{i}^{*}$ and it follows a gamma $G\left(m, \alpha_{2}\right)$ distribution.

Similarly $V^{*}=\sum_{i=1}^{m} \sum_{j=1}^{k} W_{i j}$, where $W_{i j}=\left(g_{j}+1\right)\left(-\log x_{i j}\right)^{\beta_{0}}$ and that $V^{*}$ has $G\left(m k, \alpha_{1}\right)$ distribution.

Now let $\phi\left(W_{11}, Z_{1}\right)=1, \quad W_{11}>(j+k-i) Z_{1} ;=0$, elsewhere. Note that it is an unbiased estimator for estimating $\psi\left(\alpha_{1}, \alpha_{2}\right)$. Using Lehmann-Scheffe theorem, the corresponding UMVUE $\hat{\psi}_{U}$ is obtained as, see also [7],

$$
\begin{aligned}
\hat{\psi}_{U} & =E\left(\phi\left(W_{11}, Z_{1}\right) \mid V^{*}=v^{*}, U^{*}=u^{*}\right) \\
& =P\left(W_{11}>(j+k-i) Z_{1} \mid V^{*}=v^{*}, U^{*}=u^{*}\right) \\
& =\int_{C_{0}} \int f_{W_{11} \mid V^{*}}\left(w \mid v^{*}\right) f_{Z_{1} \mid U^{*}}\left(z \mid u^{*}\right) d w d z
\end{aligned}
$$

where $C_{0}=\left\{(w, z): 0<w<v^{*}, 0<z<u^{*}, w>(j+k-i) z\right\}$ and integral is evaluated under following cases,

(i) $u^{*}(j+k-i)<v^{*}$,

(ii) $u^{*}(j+k-i)>v^{*}$ and

(iii) $u^{*}(j+k-i)=v^{*}$.

Case (i):

$$
\begin{aligned}
\hat{\psi}_{U}= & {\left[u^{*} v^{*}\right]^{-1}(m-1)(m k-1) \int_{0}^{u^{*}} \int_{z(j+k-i)}^{v^{*}} } \\
& \left(1-\frac{z}{u^{*}}\right)^{m-2}\left(1-\frac{w}{v^{*}}\right)^{m k-2} d w d z \\
= & (m-1) \int_{0}^{1}(1-t)^{m-2}(1-c t)^{m k-1} d t \\
= & \sum_{\eta=0}^{m k-1}(-c)^{\eta} \frac{\left(\begin{array}{c}
m k-1 \\
\eta
\end{array}\right)}{\left(\begin{array}{c}
m+\eta-1 \\
\eta
\end{array}\right)}, \quad c=u^{*}(j+k-i) / v^{*}, t=z / u^{*} .
\end{aligned}
$$

Case (ii)

$$
\begin{aligned}
\hat{\psi}_{U}= & {\left[u^{*} v^{*}\right]^{-1}(m-1)(m k-1) \int_{0}^{u^{*}} \int_{0}^{v^{*} /(j+k-i)} } \\
& \left(1-\frac{z}{u^{*}}\right)^{m k-2}\left(1-\frac{w}{v^{*}}\right)^{m-2} d w d z \\
= & 1-\sum_{\eta=0}^{m-1}(-1)^{\eta} \frac{\left(\begin{array}{c}
m-1 \\
\eta
\end{array}\right)}{\left(\begin{array}{c}
m k+\eta-1 \\
\eta
\end{array}\right)(c)^{\eta}}
\end{aligned}
$$

Case (iii)

$$
\begin{aligned}
\hat{\psi}_{U}= & {\left[u^{*} v^{*}\right]^{-1}(m-1)(m k-1) \int_{0}^{v^{*}} \int_{w(j+k-i)}^{u^{*}} } \\
& \left(1-\frac{z}{u^{*}}\right)^{m-2}\left(1-\frac{w}{v^{*}}\right)^{m k-2} d w d z \\
= & \frac{m-1}{m k+m-2} .
\end{aligned}
$$

The desired UMVUE of $R_{s, k}$ is now obtained as

$$
\hat{R}^{U}=\sum_{i=s}^{k} \sum_{j=0}^{i}\left[\left(\begin{array}{l}
k \\
i
\end{array}\right)\left(\begin{array}{l}
i \\
j
\end{array}\right)(-1)^{j} \hat{\psi}_{U}\right] \text {. }
$$

\subsection{Lindley's approximation to estimate sys- tem reliability $\tilde{R}^{0}$}

In this subsection, point and interval estimates of $R_{s, k}$ are evaluated using the Bayesian method when $\beta$ is known. We take the prior for $\alpha_{1}$ and $\alpha_{2}$ as $G\left(p_{1}, q_{1}\right)$ and $G\left(p_{2}, q_{2}\right)$ distributions, respectively. When $\beta=\beta_{0}$, the Bayes estimate of $R_{s, k}$ denoted by $\tilde{R}^{0}$ using Lindley's approximation method is obtained as

$$
\begin{aligned}
\tilde{R}^{0}= & u(\theta)+\left(u_{1} a_{1}+u_{2} a_{2}+a_{3}\right)+0.5\left[P_{0}\left(u_{1} \sigma_{11}+u_{2} \sigma_{12}\right)\right. \\
& \left.+Q_{0}\left(u_{1} \sigma_{21}+u_{2} \sigma_{22}\right)\right]
\end{aligned}
$$

where

$$
\begin{aligned}
a_{i} & =\rho_{1} \sigma_{i 1}+\rho_{2} \sigma_{i 2}, \quad i=1,2 . \\
a_{3} & =0.5\left(u_{11} \sigma_{11}+u_{12} \sigma_{12}+u_{21} \sigma_{21}+u_{22} \sigma_{22}\right) \\
P_{0} & =l_{111} \sigma_{11}+l_{121} \sigma_{12}+l_{211} \sigma_{21}+l_{221} \sigma_{22} . \\
Q_{0} & =l_{112} \sigma_{11}+l_{122} \sigma_{12}+l_{212} \sigma_{21}+l_{222} \sigma_{22} .
\end{aligned}
$$

Further, $\rho_{1}=\frac{p_{1}-1}{\alpha_{1}}-q_{1}, \rho_{2}=\frac{p_{2}-1}{\alpha_{2}}-q_{2}$ and $\sigma_{i j}$ is the element of the matrix associated with $\left[-l_{i j}\right]^{-1}, i, j=1,2, u(\theta)=R_{s, k}$. Each term of Bayes estimate is computed at MLEs of $\alpha_{1}$ and $\alpha_{2}$.

\subsubsection{Metropolis-Hasting algorithm}

Here we apply this method to evaluate some alternative estimate of $R_{s, k}$. We also compute credible intervals under the given censored data. We observe that posterior distributions of $\alpha_{1}, \alpha_{2}$ and $\beta$ are not in closed form. Thus it is difficult to obtain associated marginal distributions. So we approximate these marginal distributions numerically using normal proposal distributions. We apply following algorithm.

Step 1: Choose an initial guess $\left(\alpha_{1_{0}}, \alpha_{2_{0}}\right)$ of $\left(\alpha_{1}, \alpha_{2}\right)$ and set $d=1$.

Step 2: Generate $\alpha_{1(d)}$ using $N\left(\alpha_{1(d-1)}, \sigma^{2}\right)$ distribution.

Step 3: Generate $\alpha_{2(d)}$ using $N\left(\alpha_{2(d-1)}, \sigma^{2}\right)$ distribution.

Step 4: Compute $R_{(d)}=\sum_{i=s}^{k} \sum_{j=0}^{i}\left(\begin{array}{l}i \\ j\end{array}\right)\left(\begin{array}{l}k \\ i\end{array}\right)(-1)^{j} \alpha_{2(d)}\left[\alpha_{1(d)}(j+\right.$ $\left.k-i)+\alpha_{2(d)}\right]^{-1}$.

Step 5: Set $d=d+1$.

Step 6: Repeat steps 2 to $5, n_{0}$ times.

Bayes estimate of the system reliability $R_{s, k}$ is now obtained as

$$
\tilde{R}_{s, k}^{M}=\frac{1}{n_{0}} \sum_{i=1}^{n_{0}} R_{(d) s, k} .
$$

The $100(1-\eta) \%$ HPD interval of the reliability is evaluated using the method of [9].

\section{Numerical Results}

Now we examine the behavior of all estimators of multicomponent stress-strength reliability via Monte Carlo simulations on the basis of progressively censored samples. Point estimators are compared with respect to mean square error (MSE) and bias values. Similarly all interval estimators are compared 
against in terms of their average interval length and coverage probabilities (CPs). The $R$ statistical software is used for evaluating different estimates. Algorithm of [6] is used for simulation purposes. We present this algorithm for the sake of completeness.

- We first generate independent samples $Y_{1}, Y_{2}, \ldots Y_{k}$ from the standard uniform distribution.

- Consider $e_{i}=Y_{i}^{1 /\left(i+\sum_{j=k-i+1}^{k} g_{j}\right)}, i=1,2, \ldots, k$.

- Assume that $u_{i: k: K}=1-\left[e_{k} e_{k-1} \ldots e_{k-i+1}\right]$ then $e_{i: k: K}$ is progressive type II censored data from uniform distribution. Let $X_{i: k: K}=F_{X}^{-1}\left(U_{i: k: K}\right)$. Then $X_{1: k: K}, X_{2: k: K}, \ldots X_{i: k: K}$ are progressively censored samples corresponding to strength variables for a single component system. Proceeding in this way, multicomponent data can be generated. Generation of stress variables is taken care similarly.

- We mention that estimated risk values of an estimator $\hat{R}$ of reliability function $R_{s, k}$ is evaluated as $\frac{1}{n_{0}} \sum_{i=1}^{n_{0}}\left(\hat{R}_{i}-R_{s, k}\right)^{2}$ based on different effective sample sizes $n_{0}$.

In Table 1 , considered schemes $G_{1}, G_{2}, \ldots, G_{9}$ for strength variables and $F_{1}, F_{2}, \ldots, F_{9}$ for stress variables are presented. We obtain these estimates using various $(M, m, K, k)$ values. Note that $M$ represents total systems, $K$ represents total components in each system and $m$ represents the observed systems with $k$ being observed components in each system. Further $f$ is assigned values as $f=1,2$, that is, either at least one component overcomes the stress or at least two components overcome the stress of system. Estimation results are presented for both known and unknown $\beta$ cases.

$\beta$ unknown case is discussed first. Under this set up strength and stress variables are evaluated by assigning the parameters $\left(\alpha_{1}, \alpha_{2}, \beta\right)$ as $(1,0.5,2)$. In tables $2-5$ we have computed various estimated quantities for the unknown common parameter case. Different effective sample sizes are taken into consideration for evaluating the estimation results. Bayes estimates are evaluated when hyperparameters are assigned as $p_{1}=3, q_{1}=2, p_{2}=2, q_{2}=1, p_{3}=3, q_{3}=1.5$. Noninformative Bayes point and interval estimates are also evaluated against improper prior where all hyperparameters are assigned as zero. Tables 2 and 3 respectively contain point and intervals estimates of the reliability for $f=1$, while tables 4 and 5 respectively contain the similar results for $f=2$. In tables 2 and 4 , for each censoring scheme, two estimates are presented for each estimation method where first value denote the estimated risk of each method and immediate lower estimate is the corresponding bias.
Table 1: Censoring scheme for different $(M, m, K, k)$

\begin{tabular}{llllll}
\hline$(K, k)$ & & $(C, s)$ & $(M, m)$ & & $(C, s)$ \\
\hline$(10,4)$ & $G_{1}$ & $(1,3,2,0)$ & $(20,10)$ & $F_{1}$ & $\left(1^{* 10}\right)$ \\
& $G_{2}$ & $\left(6,0^{* 3}\right)$ & & $F_{2}$ & $\left(10,0^{* 9}\right)$ \\
& $G_{3}$ & $\left(0^{* 3}, 6\right)$ & & $F_{3}$ & $\left(0^{* 9}, 10\right)$ \\
$(12,5)$ & $G_{4}$ & $\left(7,0^{* 4}\right)$ & $(25,8)$ & $F_{4}$ & $\left(17,0^{* 7}\right)$ \\
& $G_{5}$ & $\left(0^{* 2}, 7,0^{* 2}\right)$ & & $F_{5}$ & $\left(0^{* 2}, 10,5,2,0^{* 3}\right)$ \\
& $G_{6}$ & $\left(0^{* 2}, 7,0^{* 2}\right)$ & & $F_{6}$ & $\left(0^{* 7}, 17\right)$ \\
$(15,11)$ & $G_{7}$ & $\left(4,0^{* 10}\right)$ & $(22,15)$ & $F_{7}$ & $\left(7,0^{* 14}\right)$ \\
& $G_{8}$ & $\left(0^{* 5}, 2,2,0^{* 4}\right)$ & & $F_{8}$ & $\left(0^{* 5}, 2,3,2,0^{* 7}\right)$ \\
& $G_{9}$ & $\left(0^{* 10}, 4\right)$ & & $F_{9}$ & $\left(0^{* 14}, 7\right)$ \\
\hline
\end{tabular}

In these tables true values of the reliability are also listed which are obtained for different effective sample sizes. From tables 2 and 4 we are able to observe that MLE of the reliability compete quite good with those of Bayesian estimates. In particular performance of MLE is quite comparable with the noninformative Bayes estimates. However proper Bayes estimates indicate better behavior than these two estimates as far as estimated risks are concerned. Performance of all estimates in terms of risk behavior get better with large sample size. In tables 3 and 5 , asymptotic, HPD and noninformative HPD (NHPD), boot$\mathrm{p}$ and boot-t intervals are presented for different sample sizes. In particular, for each censoring scheme, two values are listed for each procedure. The first one is coverage probability and lower estimate is the interval length. We see that bootstrap intervals compete good with those of asymptotic and improper HPD intervals under interval width criterion. Over all proper Bayes intervals have shortest interval length among proposed methods. Various estimates of interval length become shorter with large sample sizes. The coverage probabilities of proposed intervals are closed to the nominal level which is taken as 0.95 . Estimation results for known $\beta$ case are reported in tables 6-9 for various censoring schemes when $\beta=2$. Results of tables 6 and 8 indicate that unbiased estimate of the reliability performs quite good when compared with corresponding maximum likelihood estimate. However performance of the proper Bayes estimates is superior than the other proposed estimates. This holds true for various censoring schemes. From different intervals as listed in tables 7 and 9, we are able to observe that bootstrap intervals compete well with asymptotic intervals. Proper prior Bayes intervals yield better interval estimates than the asymptotic, noninformative Bayes and bootstrap intervals. Average length of these methods improve with sample size. The CPs of proposed intervals lie within satisfactory range of nominal level. We find that more efficient estimates of reliability can be obtained when the common parameter is considered as known. Here we assess the efficiency of various point estimates using MSE values where as efficiency of intervals are measured under average width criterion. 
Table 2: Point estimates of $R_{s, k}$ when $\left(\alpha_{1}, \alpha_{2}, \beta\right)=(1,0.5,2)$ and $\beta$ is unknown

\begin{tabular}{|c|c|c|c|c|c|c|c|}
\hline$(M, m, K, k)$ & $C S$ & $R_{t}$ & $\begin{array}{r}\hat{R}^{M L} \\
\quad f=\end{array}$ & $\hat{R}^{M H}$ & $\hat{R}^{L I N}$ & $\hat{R}^{N M H}$ & $\hat{R}^{N L I N}$ \\
\hline \multirow[t]{6}{*}{$(20,10,10,4)$} & $\left(G_{1}, F_{1}\right)$ & 0.88888 & 0.00456 & 0.00276 & 0.00283 & 0.00294 & 0.00297 \\
\hline & & & -0.03981 & -0.01397 & -0.01934 & 0.00148 & -0.02202 \\
\hline & $\left(G_{2}, F_{2}\right)$ & & 0.00394 & 0.00258 & 0.00275 & 0.00272 & 0.00305 \\
\hline & & & -0.03733 & -0.01297 & -0.01446 & -0.01318 & -0.01750 \\
\hline & $\left(G_{3}, F_{3}\right)$ & & 0.00382 & 0.00230 & 0.00257 & 0.00265 & 0.00270 \\
\hline & & & -0.03070 & -0.01068 & -0.01266 & -0.01357 & 0.01505 \\
\hline \multirow[t]{6}{*}{$(25,8,12,5)$} & $\left(G_{4}, F_{4}\right)$ & 0.90909 & 0.00252 & 0.00143 & 0.00151 & 0.00199 & 0.00187 \\
\hline & & & -0.02359 & -0.01007 & -0.01329 & -0.01573 & 0.01899 \\
\hline & $\left(G_{5}, F_{5}\right)$ & & 0.00246 & 0.00156 & 0.00232 & 0.00165 & 0.00190 \\
\hline & & & -0.02064 & 0.00994 & -0.01183 & 0.01116 & -0.01368 \\
\hline & $\left(G_{6}, F_{6}\right)$ & & 0.00199 & 0.00137 & 0.00159 & 0.00154 & 0.00175 \\
\hline & & & 0.01141 & -0.01006 & -0.01108 & 0.01101 & 0.01251 \\
\hline \multirow[t]{6}{*}{$(22,15,15,11)$} & $\left(G_{7}, F_{7}\right)$ & 0.95652 & 0.00115 & 0.00032 & 0.00046 & 0.00098 & 0.00090 \\
\hline & & & -0.00948 & -0.00620 & -0.00897 & -0.01263 & -0.01212 \\
\hline & $\left(G_{8}, F_{8}\right)$ & & 0.00102 & 0.00026 & 0.00038 & 0.00104 & 0.00081 \\
\hline & & & -0.00898 & -0.00536 & 0.00756 & -0.01015 & -0.00989 \\
\hline & $\left(G_{9}, F_{9}\right)$ & & 0.00091 & 0.00026 & 0.00013 & 0.00092 & 0.00063 \\
\hline & & & -0.00725 & -0.00394 & -0.00512 & 0.00079 & -0.00823 \\
\hline
\end{tabular}

Table 3: Interval estimates of $R_{s, k}$ when $\left(\alpha_{1}, \alpha_{2}, \beta\right)=(1,0.5,2)$ and $\beta$ is unknown

\begin{tabular}{cccccccc}
\hline$(M, m, K, k)$ & $C S$ & $R_{t}$ & $A S$ & $H P D$ & $N H P D$ & Boot $-p$ & Boot $-t$ \\
\hline$(20,10,10,4)$ & $\left(G_{1}, F_{1}\right)$ & 0.88888 & 0.905 & 0.946 & 0.941 & 0.915 & 0.930 \\
& & & 0.15301 & 0.13134 & 0.13354 & 0.17079 & 0.18278 \\
& $\left(G_{2}, F_{2}\right)$ & & 0.919 & 0.942 & 0.939 & 0.899 & 0.901 \\
& & & 0.15556 & 0.13810 & 0.13965 & 0.17398 & 0.18642 \\
& $\left(G_{3}, F_{3}\right)$ & & 0.924 & 0.958 & 0.956 & 0.944 & 0.948 \\
$(25,8,12,5)$ & & & 0.14299 & 0.12941 & 0.12945 & 0.16226 & 0.17652 \\
& $\left(G_{4}, F_{4}\right)$ & 0.90909 & 0.912 & 0.965 & 0.952 & 0.936 & 0.940 \\
& & & 0.13662 & 0.11235 & 0.11443 & 0.15492 & 0.16486 \\
& $\left(G_{5}, F_{5}\right)$ & & 0.904 & 0.962 & 0.953 & 0.935 & 0.938 \\
& & & 0.13290 & 0.11375 & 0.11527 & 0.15871 & 0.17028 \\
$\left(G_{6}, F_{6}\right)$ & & 0.921 & 0.968 & 0.951 & 0.947 & 0.949 \\
& & & 0.13028 & 0.11076 & 0.11138 & 0.15425 & 0.16332 \\
& $\left(G_{7}, F_{7}\right)$ & 0.95652 & 0.938 & 0.957 & 0.954 & 0.942 & 0.956 \\
& & & 0.07518 & 0.04680 & 0.04694 & 0.10523 & 0.11278 \\
& $\left(G_{8}, F_{8}\right)$ & & 0.945 & 0.955 & 0.951 & 0.939 & 0.948 \\
& & & 0.07412 & 0.04303 & 0.04314 & 0.09719 & 0.10485 \\
& $\left(G_{9}, F_{9}\right)$ & & 0.948 & 0.967 & 0.966 & 0.942 & 0.956 \\
& & & 0.06875 & 0.04477 & 0.04385 & 0.09548 & 0.10276 \\
\hline
\end{tabular}

Table 4: Point estimates of $R_{s, k}$ when $\left(\alpha_{1}, \alpha_{2}, \beta\right)=(1,0.5,2)$ and $\beta$ is unknown

\begin{tabular}{cccccccc}
\hline$(M, m, K, k)$ & $C S$ & $R_{t}$ & $\hat{R}^{M L}$ & $\hat{R}^{M H}$ & $\hat{R}^{L I N}$ & $\hat{R}^{N M H}$ & $\hat{R}^{N L I N}$ \\
\hline$(20,10,10,4)$ & $\left(G_{1}, F_{1}\right)$ & 0.76190 & 0.01062 & 0.00889 & 0.00892 & 0.00893 & 0.00897 \\
& & & -0.04620 & -0.01902 & -0.02230 & 0.00205 & -0.02362 \\
& $\left(G_{2}, F_{2}\right)$ & & 0.00978 & 0.00843 & 0.00854 & 0.00855 & 0.00864 \\
& & & -0.04309 & -0.01809 & -0.02130 & -0.01951 & -0.02159 \\
& $\left(G_{3}, F_{3}\right)$ & & 0.01030 & 0.00840 & 0.00851 & 0.00955 & 0.00861 \\
$(25,8,12,5)$ & & & -0.03981 & -0.01746 & -0.01980 & -0.01841 & -0.02041 \\
& $\left(G_{4}, F_{4}\right)$ & 0.80808 & 0.00841 & 0.00637 & 0.00691 & 0.00720 & 0.00723 \\
& & & -0.03534 & -0.01458 & -0.01669 & -0.01518 & -0.01785 \\
& $\left(G_{5}, F_{5}\right)$ & & 0.00836 & 0.00664 & 0.00672 & 0.00745 & 0.00766 \\
& & & -0.03589 & 0.01497 & -0.01672 & 0.01553 & -0.01792 \\
$\left(G_{6}, F_{6}\right)$ & & 0.00812 & 0.00629 & 0.00650 & 0.00722 & 0.00720 \\
& & & -0.03436 & -0.01420 & 0.01611 & 0.01508 & 0.01723 \\
& $\left(G_{7}, F_{7}\right)$ & 0.91097 & 0.00562 & 0.00327 & 0.00346 & 0.00403 & 0.00410 \\
& & & -0.01890 & -0.00516 & -0.00696 & -0.00551 & -0.00745 \\
& $\left(G_{8}, F_{8}\right)$ & & 0.00591 & 0.00334 & 0.00360 & 0.00413 & 0.00416 \\
& & & -0.01898 & -0.00527 & -0.00705 & 0.00601 & 0.00764 \\
& $\left(G_{9}, F_{9}\right)$ & & 0.00540 & 0.00294 & 0.00316 & 0.00387 & 0.00390 \\
& & & -0.01717 & -0.00505 & 0.00705 & 0.00531 & -0.00730 \\
\hline
\end{tabular}

Table 5: Interval estimates of $R_{s, k}$ when $\left(\alpha_{1}, \alpha_{2}, \beta\right)=(1,0.5,2)$ and $\beta$ is unknown

\begin{tabular}{cccccccc}
\hline$(M, m, K, k)$ & $C S$ & $R_{t}$ & $A S$ & $H P D$ & $N H P D$ & Boot $-p$ & Boot $-t$ \\
\hline$(20,10,10,4)$ & $\left(G_{1}, F_{1}\right)$ & 0.76190 & 0.915 & 0.951 & 0.957 & 0.925 & 0.940 \\
& & & 0.27014 & 0.25333 & 0.25657 & 0.30268 & 0.32643 \\
& $\left(G_{2}, F_{2}\right)$ & & 0.921 & 0.957 & 0.946 & 0.939 & 0.920 \\
& & & 0.27240 & 0.25139 & 0.25484 & 0.30094 & 0.31996 \\
$\left(G_{3}, F_{3}\right)$ & & 0.926 & 0.964 & 0.954 & 0.949 & 0.951 \\
$(25,8,12,5)$ & & & 0.26980 & 0.25017 & 0.25180 & 0.29596 & 0.30297 \\
& $\left(G_{4}, F_{4}\right)$ & 0.80808 & 0.929 & 0.966 & 0.965 & 0.947 & 0.940 \\
& & & 0.24917 & 0.22851 & 0.23027 & 0.27888 & 0.28943 \\
& $\left(G_{5}, F_{5}\right)$ & & 0.918 & 0.963 & 0.965 & 0.941 & 0.940 \\
& & & 0.24896 & 0.22722 & 0.22711 & 0.27819 & 0.28820 \\
$\left(G_{6}, F_{6}\right)$ & & 0.932 & 0.968 & 0.962 & 0.945 & 0.950 \\
& & & 0.22901 & 0.22561 & 0.22606 & 0.27491 & 0.28491 \\
& $\left(G_{7}, F_{7}\right)$ & 0.91097 & 0.945 & 0.968 & 0.962 & 0.949 & 0.951 \\
& & & 0.12957 & 0.09214 & 0.09470 & 0.14921 & 0.15640 \\
& $\left(G_{8}, F_{8}\right)$ & & 0.943 & 0.957 & 0.956 & 0.949 & 0.955 \\
& & & 0.12674 & 0.09469 & 0.09513 & 0.14419 & 0.15722 \\
& $\left(G_{9}, F_{9}\right)$ & & 0.946 & 0.969 & 0.964 & 0.945 & 0.950 \\
& & & 0.11534 & 0.08967 & 0.09077 & 0.14027 & 0.14677 \\
\hline
\end{tabular}

Table 6: Point estimates of $R_{s, k}$ when $\left(\alpha_{1}, \alpha_{2}, \beta\right)=(1,0.5,2)$ and $\beta$ is known

\begin{tabular}{|c|c|c|c|c|c|c|c|c|}
\hline$(M, m, K, k)$ & $C S$ & $R_{t}$ & $\hat{R}^{M L}$ & $\begin{array}{c}\hat{R}^{U M V} \\
f=1\end{array}$ & $\hat{R}^{M H}$ & $\hat{R}^{L I N}$ & $\hat{R}^{N M H}$ & $\hat{R}^{N L I N}$ \\
\hline \multirow[t]{6}{*}{$(20,10,10,4)$} & $\left(G_{1}, F_{1}\right)$ & 0.88888 & 0.00512 & 0.00436 & 0.00244 & 0.00341 & 0.00293 & 0.00395 \\
\hline & & & & -0.02100 & -0.02209 & -0.02673 & & -0.02908 \\
\hline & $\left(G_{2}, F_{2}\right)$ & & 0.00524 & 0.00448 & 0.00255 & 0.00351 & 0.00294 & 0.00416 \\
\hline & & & -0.04206 & -0.02151 & -0.02291 & -0.02662 & -0.02585 & -0.03097 \\
\hline & $\left(G_{3}, F_{3}\right)$ & & 0.00502 & 0.00418 & 0.00234 & 0.00323 & 0.00285 & 0.00382 \\
\hline & & & -0.04024 & -0.02092 & -0.02189 & -0.02553 & -0.02337 & 0.02812 \\
\hline \multirow[t]{6}{*}{$(25,8,12,5)$} & $\left(G_{4}, F_{4}\right)$ & 0.90909 & 0.00352 & 0.00360 & 0.00185 & 0.00269 & 0.00227 & 0.00305 \\
\hline & & & -0.03147 & 0.01650 & -0.01683 & -0.01930 & -0.01861 & -0.02171 \\
\hline & $\left(G_{5}, F_{5}\right)$ & & 0.00364 & 0.00372 & 0.00191 & 0.00289 & 0.00241 & 0.00320 \\
\hline & & & -0.03259 & 0.01685 & 0.01707 & -0.01981 & -0.01854 & -0.02194 \\
\hline & $\left(G_{6}\right.$ & & 0.00343 & 0.00337 & 0.00160 & 0.00 & 0.00219 & 0.00285 \\
\hline & & & -0.02 & 0.01593 & & -0.0 & -0.0 & -0.02034 \\
\hline \multirow[t]{6}{*}{$(22,15,15,11)$} & $\left(G_{7}, F_{7}\right)$ & 0.95652 & 0.00095 & 0.00237 & 0.00 & 0.00 & 0.00084 & 0.00107 \\
\hline & & & -0.01226 & 0.00708 & -0.00724 & -0.00955 & -0.00759 & -0.00992 \\
\hline & $\left(G_{8}\right.$, & & 0.00103 & 0.00141 & 0.00055 & 0.00079 & 0.00099 & 0.00011 \\
\hline & & & -0.0 & 0.00 & -0.0 & -0.0 & & -0.01008 \\
\hline & $\left(G_{9}, F_{9}\right)$ & & 0.00 & 0.00130 & 0.00042 & 0.00063 & 0.0 & 0.00092 \\
\hline & & & -0.01171 & 0.00651 & 0.00717 & -0.00900 & -0.00740 & -0.00941 \\
\hline
\end{tabular}

Table 7: Interval estimates of $R_{s, k}$ when $\left(\alpha_{1}, \alpha_{2}, \beta\right)=(1,0.5,2)$ and $\beta$ is known

\begin{tabular}{cccccccc}
\hline$(M, m, K, k)$ & $C S$ & $R_{t}$ & $A S$ & $H P D$ & NHPD & Boot $-p$ & Boot $-t$ \\
& & & $f=1$ & & & & \\
\hline$(20,10,10,4)$ & $\left(G_{1}, F_{1}\right)$ & 0.88888 & 0.919 & 0.952 & 0.955 & 0.927 & 0.940 \\
& & & 0.14183 & 0.12689 & 0.12770 & 0.16183 & 0.17568 \\
& $\left(G_{2}, F_{2}\right)$ & & 0.923 & 0.956 & 0.946 & 0.936 & 0.930 \\
& & & 0.14137 & 0.12807 & 0.12881 & 0.16441 & 0.17675 \\
$\left(G_{3}, F_{3}\right)$ & & 0.929 & 0.962 & 0.951 & 0.948 & 0.950 \\
& & & 0.14013 & 0.12489 & 0.12593 & 0.16094 & 0.17354 \\
& $\left(G_{4}, F_{4}\right)$ & 0.90909 & 0.927 & 0.967 & 0.969 & 0.947 & 0.942 \\
& & & 0.12713 & 0.10411 & 0.10678 & 0.14116 & 0.15516 \\
& $\left(G_{5}, F_{5}\right)$ & & 0.929 & 0.967 & 0.966 & 0.942 & 0.940 \\
& & & 0.12628 & 0.10305 & 0.10470 & 0.14128 & 0.15368 \\
$\left(G_{6}, F_{6}\right)$ & & 0.934 & 0.963 & 0.965 & 0.946 & 0.950 \\
& & & 0.12414 & 0.10149 & 0.10158 & 0.14094 & 0.14992 \\
& $\left(G_{7}, F_{7}\right)$ & 0.95652 & 0.948 & 0.963 & 0.965 & 0.944 & 0.950 \\
& & & 0.06415 & 0.04509 & 0.04716 & 0.08999 & 0.09181 \\
& $\left(G_{8}, F_{8}\right)$ & & 0.946 & 0.959 & 0.955 & 0.943 & 0.953 \\
& & & 0.06658 & 0.04548 & 0.04606 & 0.08832 & 0.09084 \\
& $\left(G_{9}, F_{9}\right)$ & & 0.940 & 0.962 & 0.965 & 0.942 & 0.950 \\
& & & 0.06479 & 0.04322 & 0.04516 & 0.08803 & 0.08960 \\
\hline
\end{tabular}

Table 8: Point estimates of $R_{s, k}$ when $\left(\alpha_{1}, \alpha_{2}, \beta\right)=(1,0.5,2)$ and $\beta$ is known

\begin{tabular}{|c|c|c|c|c|c|c|c|c|}
\hline$(M, m, K, k)$ & $C S$ & $R_{t}$ & $\hat{R}^{M L}$ & $\begin{array}{c}\hat{R}^{U M V} \\
f=2\end{array}$ & $\hat{R}^{M H}$ & $\hat{R}^{L I N}$ & $\hat{R}^{N M H}$ & $\hat{R}^{N L I N}$ \\
\hline \multirow[t]{6}{*}{$(20,10,10,4)$} & $\left(G_{1}, F_{1}\right)$ & 0.76190 & 0.01072 & 0.00913 & 0.00835 & 0.00891 & 0.00956 & 0.00988 \\
\hline & & & -0.04460 & 0.01958 & -0.01980 & -0.02200 & -0.02017 & -0.02274 \\
\hline & $\left(G_{2}, F_{2}\right)$ & & 0.00949 & 0.00937 & 0.00854 & 0.00899 & 0.00969 & 0.00987 \\
\hline & & & -0.04560 & 0.01973 & -0.01991 & -0.2221 & -0.02047 & -0.02308 \\
\hline & $\left(G_{3}, F_{3}\right)$ & & 0.00948 & 0.00908 & 0.00817 & 0.00874 & 0.00939 & 0.00923 \\
\hline & & & -0.04385 & 0.01930 & -0.01924 & -0.02139 & -0.02008 & -0.02258 \\
\hline \multirow[t]{6}{*}{$(25,8,12,5)$} & $\left(G_{4}, F_{4}\right)$ & 0.80808 & 0.00810 & 0.00873 & 0.00689 & 0.00709 & 0.00748 & 0.00784 \\
\hline & & & -0.02714 & 0.01104 & -0.01121 & -0.01252 & -0.01175 & -0.01308 \\
\hline & $\left(G_{5}, F_{5}\right)$ & & 0.00816 & 0.00888 & 0.00694 & 0.00716 & 0.00768 & 0.00802 \\
\hline & & & -0.02781 & 0.01182 & 0.01194 & -0.1296 & -0.01206 & -0.01329 \\
\hline & $\left(G_{6}, F_{6}\right)$ & & 0.00765 & 0.00868 & 0.00575 & 0.00613 & 0.00698 & 0.00721 \\
\hline & & & -0.02666 & 0.01072 & 0.01103 & -0.01223 & -0.01150 & -0.01284 \\
\hline \multirow[t]{6}{*}{$(22,15,15,11)$} & $\left(G_{7}, F_{7}\right)$ & 0.91097 & 0.00561 & 0.00465 & 0.00278 & 0.00297 & 0.00327 & 0.00332 \\
\hline & & & -0.01281 & 0.00545 & -0.00578 & -0.00629 & -0.00601 & -0.00671 \\
\hline & $\left(G_{8}, F_{8}\right)$ & & 0.00584 & 0.00375 & 0.00292 & 0.00302 & 0.00334 & 0.00345 \\
\hline & & & -0.01298 & 0.00564 & -0.00591 & -0.00638 & -0.00615 & -0.00698 \\
\hline & $\left(G_{9}, F_{9}\right)$ & & 0.00541 & 0.00342 & 0.00257 & 0.00289 & 0.00311 & 0.00319 \\
\hline & & & -0.01258 & 0.00519 & 0.00571 & -0.00609 & -0.00593 & 0.00651 \\
\hline
\end{tabular}

Table 9: Interval estimates of $R_{s, k}$ when $\left(\alpha_{1}, \alpha_{2}, \beta\right)=(1,0.5,2)$ and $\beta$ is known

\begin{tabular}{|c|c|c|c|c|c|c|c|}
\hline$(M, m, K, k)$ & $C S$ & $R_{t}$ & $\begin{array}{l}A S \\
f=\end{array}$ & $H P D$ & $N H P D$ & Boot $-p$ & Boot $-t$ \\
\hline \multirow[t]{6}{*}{$(20,10,10,4)$} & $\left(G_{1}, F_{1}\right)$ & 0.76190 & 0.912 & 0.957 & 0.959 & 0.934 & 0.942 \\
\hline & & & 0.25052 & 0.23220 & 0.23390 & 0.28390 & 0.29528 \\
\hline & $\left(G_{2}, F_{2}\right)$ & & 0.821 & 0.957 & 0.942 & 0.947 & 0.942 \\
\hline & & & 0.25089 & 0.23358 & 0.23413 & 0.28518 & 0.29979 \\
\hline & $\left(G_{3}, F_{3}\right)$ & & 0.929 & 0.959 & 0.953 & 0.947 & 0.952 \\
\hline & & & 0.24991 & 0.23067 & 0.23062 & 0.28211 & 0.29546 \\
\hline \multirow[t]{6}{*}{$(25,8,12,5)$} & $\left(G_{4}, F_{4}\right)$ & 0.80808 & 0.927 & 0.963 & 0.962 & 0.946 & 0.951 \\
\hline & & & 0.22863 & 0.20215 & 0.20497 & 0.25766 & 0.26489 \\
\hline & $\left(G_{5}, F_{5}\right)$ & & 0.934 & 0.966 & 0.964 & 0.941 & 0.953 \\
\hline & & & 0.23053 & 0.20338 & 0.20788 & 0.25745 & 0.26244 \\
\hline & $\left(G_{6}, F_{6}\right)$ & & 0.935 & 0.965 & 0.959 & 0.945 & 0.952 \\
\hline & & & 0.22566 & 0.20094 & 0.20172 & 0.25582 & 0.26005 \\
\hline \multirow[t]{6}{*}{$(22,15,15,11)$} & $\left(G_{7}, F_{7}\right)$ & 0.91097 & 0.944 & 0.959 & 0.951 & 0.949 & 0.950 \\
\hline & & & 0.10784 & 0.08843 & 0.09086 & 0.12920 & 0.13506 \\
\hline & $\left(G_{8}, F_{8}\right)$ & & 0.947 & 0.961 & 0.957 & 0.942 & 0.948 \\
\hline & & & 0.10841 & 0.08892 & 0.09090 & 0.13201 & 0.13787 \\
\hline & $\left(G_{9}, F_{9}\right)$ & & 0.951 & 0.963 & 0.964 & 0.94 & 0.953 \\
\hline & & & 0.10544 & 0.08801 & 0.08933 & 0.13014 & 0.13643 \\
\hline
\end{tabular}


Table 10: Goodness of fit results for the real data

\begin{tabular}{|c|c|c|c|c|c|c|c|c|}
\hline \multirow[t]{2}{*}{ Distribution } & \multicolumn{4}{|l|}{ Data Z } & \multicolumn{4}{|l|}{ Data $\mathrm{T}$} \\
\hline & & $\beta$ & K-S & p-value & $\hat{\alpha}_{2}$ & $\bar{\beta}$ & K-S & p-value \\
\hline Unit & 0.6440496 & 1.66 & 0.11066 & 0.8866 & 0.223873 & 2.182262 & 0.30851 & 0.6304 \\
\hline Log-I & 0.8732852 & 99.4856 & 0.20042 & 0.2343 & 0.5787747 & 83.40 & 0.41813 & 0.2634 \\
\hline Kumarswamy & 1.486776 & 2.636823 & 0.12046 & 0.8196 & 1.080441 & 3.371279 & 0.35603 & 0.4477 \\
\hline Unit-Gamma & 1252170 & 0.86346 & 0.20044 & 0.2341 & 1004119 & 0.5672637 & 0.4182 & 0.2632 \\
\hline
\end{tabular}

\section{Data analysis}

Now we discuss application of proposed methods using one numerical example. The considered data come from Xia et al. (2009) which defines jute fiber strength at various gauge lengths. The associated diameters of fibers are measured using an XSP8CA biological microscope (Shanghai Optical Instrument Factory, Shanghai, China). The data are given as follows:

693.73, 704.66, 323.83, 778.17, 123.06, 637.66, 383.43, 151.48, $108.94,50.16,671.49,183.16,257.44,727.23,291.27,101.15$, 376.42 , 163.40, 141.38, 700.74, 262.90, 353.24, 422.11, 43.93, $590.48,212.13,303.90,506.60,530.55,177.25$.

Here, we consider $s=1,2$ and $k=3$. This indicates a 1 or 2-out-of-4: $\mathrm{G}$ system. If $T_{1}$ be the sixth failure time and $X_{1 k}, k=1,2, \cdots, 3$, be the failure times of observations numbered from 1 to 5 . Similarly, let $T_{2}$ be the failure time of the 12th observations and $X_{2 k}, k=1,2, \cdots, 3$, be the failure times of observations lying between 7 to 11 . We carried out this data process up to 30th failure and $m=4$ data are obtained for $T$. The observed data $(Z, T)$ are as follows:

The data set under Scheme 1 is as follows:

$$
X=\left[\begin{array}{lll}
0.10894 & 0.05016 & 0.67149 \\
0.29127 & 0.10115 & 0.37642 \\
0.26290 & 0.35324 & 0.42211 \\
0.30390 & 0.50660 & 0.53055
\end{array}\right] T=\left[\begin{array}{l}
0.18316 \\
0.16340 \\
0.04393 \\
0.17725
\end{array}\right]
$$

The data set under Scheme 2 is as follows:

$$
X=\left[\begin{array}{lll}
0.69373 & 0.70466 & 0.32383 \\
0.38343 & 0.15148 & 0.10894 \\
0.25744 & 0.72723 & 0.29127 \\
0.14138 & 0.70074 & 0.26290
\end{array}\right] T=\left[\begin{array}{l}
0.63766 \\
0.18316 \\
0.16340 \\
0.04393
\end{array}\right]
$$

Each data value is divided by 1000 for computation purposes. Then we verify if the data originate from the two parameter unit-Weibull distribution. The goodness-of-fit test is used to estimate the model fitting and in process Log-Lindley, Kumaraswamy, and unit-Gamma distributions are also fitted. The method of maximum likelihood is applied to estimate unknown parameters. These ML estimates and KolmogorovSmirnov(KS) values with corresponding p-values are presented in Table 10. Quantities listed in Table 10 indicate that unitWeibull provides quite good fit to the fibre strength data. Tables 11-14 contain ML and Bayes estimates of $R_{s, k}$ along with asymptotic, bootstrap, and noninformative HPD intervals. The intervals are computed under $95 \%$ level. The Bayes estimates are evaluated under improper priors. The associated censoring schemes are as follows:

Scheme 1: $\mathrm{G}=(2,0,0), \mathrm{S}=(1,0,0,0)(\mathrm{M}=7, \mathrm{~K}=4, \mathrm{~m}=$ $5, \mathrm{k}=3, \mathrm{~s}=1)$.

Scheme 2: $\mathrm{G}=(0,0,2), \mathrm{S}=(0,0,0,1)(\mathrm{M}=7, \mathrm{~K}=4, \mathrm{~m}=$ $4, \mathrm{k}=2, \mathrm{~s}=2)$. From these tables, we observe that the HPD interval is having shorter length compared to the asymptotic interval.

Table 11: Point estimates of $R_{s, k}$ for the censoring scheme 1 for the real data

\begin{tabular}{llllll}
\hline$\alpha_{1}$ & $\alpha_{2}$ & $\beta$ & $R_{s, k}$ & $R_{s, k}^{M H}$ & $R_{s, k}^{L I N}$ \\
\hline 0.7095155 & 0.4265645 & 1.6203941 & 0.8330544 & 0.8526942 & 0.8373805 \\
\hline
\end{tabular}

Table 12: Interval estimates of $\boldsymbol{R}_{\boldsymbol{s}, \boldsymbol{k}}$ for the censoring scheme 1 for the real data

\begin{tabular}{llll}
\hline ACI & Boot-t & Boot-p & HPD \\
\hline$(0.6879487,0.9781602)$ & $(0.6052020 .987828)$ & $(0.6049240,0.9637762)$ & $(0.6818500,0.9578989)$ \\
\hline
\end{tabular}

Table 13: Point estimates of $\boldsymbol{R}_{\boldsymbol{s}, k}$ for the censoring scheme 2 for the real data

\begin{tabular}{llllll}
\hline$\alpha_{1}$ & $\alpha_{2}$ & $\beta$ & $R_{s, k}^{\wedge}$ & $R_{s, k}^{M H}$ & $R_{s, k}^{L I N}$ \\
\hline 0.7095155 & 0.4265645 & 1.6203941 & 0.6405141 & 0.6786757 & 0.642972 \\
\hline
\end{tabular}

Table 14: Interval estimates of $R_{s, k}$ for the censoring scheme 2 for the real data

\begin{tabular}{llll}
\hline ACI & Boot-t & Boot-p & HPD \\
\hline$(0.3744870,0.9065412)$ & $(0.28756445,0.92315481)$ & $(0.2959196,0.9051141)$ & $(0.3859285,0.8926442)$ \\
\hline
\end{tabular}

\section{Conclusions}

In this paper, inference for the stress-strength reliability is derived assuming that both stress-strength component follow unit Weibull distributions. The data are observed from progressive type II censoring. We illustrated all the estimation methods using jute fibre strength data set. Different approaches have been applied to obtain the reliability of system based on different sample sizes. We studied their performance through MSEs and biases when the common shape parameter $\beta$ is known and unknown. Further, when this common shape parameter $\beta$ is known or unknown, the Bayes estimates of $R_{s, k}$ are obtained by using both Lindley's approximation and $\mathrm{MH}$ methods. We have also obtained uniformly minimum variance unbiased estimates of $R_{s, k}$ with known shape parameter $\beta$. We have also compared the confidence intervals based on asymptotic distribution of the MLEs. The boot-p, boot-t and HPD credible intervals are obtained as well and results 
indicate good coverage probabilities. The interval length of proper HPD method remain smaller than associate asymptotic and bootstrap intervals. We observe that Bayes method yields better inference results compared to MLE and UMVUE. It is also observed that boot-p confidence intervals perform better than the Boot-t confidence intervals in terms of average length while Boot-t performs better than Boot-p in terms CPs. Through jute fibre example it is observed asymptotic interval is wider than improper HPD interval. Making inference upon reliability when stress-strength are dependent and not having the same distributions is an interesting problem. This work has the potential to be applied in the context of reliability theory and censored data analysis. Besides, we can extend this study to the progressive hybrid and adaptive progressive hybrid censored unit Weibull distribution.

\section{Acknowledgments}

This work was funded by Deanship of Scientific Research at Princess Nourah bint Abdulrahman University, through the Program of Research Project(Grant No. 41-PRFA-P-14). The authors are thankful to anonymous three referees for their useful comments and suggestions that improved the quality of the paper..

\section{References}

[1] Alsayed, A. and Manzi, G., A comparison of monotonic correlation measures with outliers, WSEAS Transactions on Computers, 18, Art. 29, (2019), 223-230.

[2] Alotaibi, R. M., Tripathi, Y. M., Dey, S. and Rezk, H. R., Bayesian and non-Bayesian reliability estimation of multicomponent stress-strength model for unit Weibull distribution, Journal of Taibah University for Science, 14(1), (2020), 1164-1181.

[3] Balakrishnan, N. and Aggarwala, R., Progressive Censoring: Theory, Methods, and Applications, (2000), Birkhauser, Boston.

[4] Balakrishnan, N., Progressive censoring methodology: An appraisal (with discussion), Test 16, (2007), pp. 211-296.

[5] Balakrishnan, N., Kannan, N., Lin, C.T. and Wu S. J., Inference for the extreme value distribution under progressive type-II censoring, Journal of Statistical Computation and Simulation, 74, (2004), pp. 25-45.

[6] Balakrishnan, N. and Sandhu., R. A., A simple simulational algorithm for generating progressive Type-II censored samples, American Statistician, 49(2), (1995), 229-230.
[7] Basirat, M., Baratpour, S. and Ahmadi, J., Statistical inferences for stress-strength in the proportional hazard models based on progressive type-ii censored samples, Journal of Statistical Computation and Simulation, 85(3), (2015), 431449 .

[8] Bhattacharyya, G. and Johnson, R. A., Estimation of reliability in a multicomponent stress-strength model, Journal of the American Statistical Association, 69(348), (1974), 966970 .

[9] Chen, M.-H. and Shao, Q.-M., Monte carlo estimation of bayesian credible and hpd intervals, Journal of Computational and Graphical Statistics, 8(1), (1999), 69-92.

[10] Cohen, A.C., Progressively censored sample in life testing, Technometrics 5, (1963), pp. 327-339.

[11] Cohen, A.C. and N.J. Norgaard, Progressively censored sampling in the three parameter gamma distribution, Technometrics 19, (1977), pp. 333-340.

[12] Dey, S., Mazucheli, J. and Anis, M., Estimation of reliability of multicomponent stress-strength for a kumaraswamy distribution, Communications in StatisticsTheory and Methods, 46, (2017), 1560-1572.

[13] Dey, S., and Moala, F. A., Estimation of reliability of multicomponent stress-strength of a bathtub shape or increasing failure rate function, International Journal of Quality $\&$ Reliability Management, 36(2), (2019), 122-136.

[14] Efron, B., The jackknife, the Bootstrap, and Other Resampling Plans, Siam, Philadelphia, 1982.

[15] Hall, P., On some simple estimates of an exponent of regular variation, Journal of the Royal Statistical Society: Series $B$ (Methodological), 1982; 44: 37-42.

[16] Hastings, W. K., Monte carlo sampling methods using markov chains and their applications, Biometrika, 57(1), (1970), 97-109.

[17] Jha, M. K., Dey, S., Alotaibi, R. M. and Tripathi, Y. M., Reliability estimation of a multicomponent stress-strength model for unit Gompertz distribution under progressive type II censoring, Quality and Reliability Engineering International, (2020), https://doi.org/10.1002/qre.2610.

[18] Jindrova, P. and Pacakova, V., Natural catastrophe models for insurance risk management, WSEAS Transactions on Business and Economics, 16, Art. 2, (2019), 9-17.

[19] Kayal, T., Tripathi, Y. M., Dey, S. and Wu, S.-J., On estimating the reliability in a multicomponent stress-strength 
model based on Chen distribution, Communications in Statistics-Theory and Methods, 49(10), (2020), 2429-2447.

[20] Kizilaslan, F., Classical and Bayesian estimation of reliability in a multicomponent stress-strength model based on a general class of inverse exponentiated distributions, Statistical Papers 59(3), (2018), 1161-1192.

[21] Kizilaslan, F. and Nadar, M., Classical and Bayesian estimation of reliability in multicomponent stress-strength model based on Weibull distribution, Revista Colombiana de Estadstica, 38(2), (2015), 67-484.

[22] Kohansal, A., On estimation of reliability in a multicomponent stress-strength model for a Kumaraswamy distribution based on progressively censored sample, Statistical Papers, 60, (2019), 2185-2224.

[23] Kumaraswamy, P., A generalized probability density function for double-bounded random processes, Journal of Hydrology, 46(1-2), (1980), 79-88.

[24] C.T. Lin, S.J.S. Wu, and N. Balakrishnan, Inference for log-gamma distribution based on progressively type-II censored data, Communications in Statistics - Theory and Methods, 35, (2006), pp. 1271-1292.

[25] Lindley, D. V., Approximate bayesian methods, Trabajos de estadstica y de investigacion operativa, 31(1), (1980), $223-245$.

[26] Maurya, R. K. and Tripathi, Y. M., Reliability estimation in a multicomponent stress-strength model for Burr XII distribution under progressive censoring, Brazilian Journal of Probability and Statistics, Vol. 34(2), (2020), 345-369.

[27] M.T. Madi and M.Z. Raqab, Bayesian inference for the generalized exponential distribution based on progressively censored data, Communications in Statistics - Theory and Methods, 38, (2009), pp. 2016-2029.

[28] Mazucheli, J., Menezes, A. F. B., and Dey, S., Improved maximum-likelihood estimators for the parameters of the unit-gamma distribution, Communications in Statistics Theory and Methods, 47(15), (2018a), 3767-3778.

[29] Mazucheli, J., Menezes, A.F.B. and Dey, S., The unitBirnbaum-Saunders distribution with applications, Chilean Journal of Statistics, 9(1), (2018b), 47-57.

[30] Mazucheli, J., Menezes, A.F.B. and Ghitany, M.E., The unit-Weibull distribution and associated inference, Journal of Applied Probability and Statistics, 13(2), (2018), 1-22.
[31] Mazucheli, J., Menezes, A.F.B. and Dey, S., Unit-Gompertz distribution with applications, Statistica, 79(1), (2019), 2543.

[32] Metropolis, N., Rosenbluth, A. W., Rosenbluth, M. N., Teller, A. H., and Teller, E., Equation of state calculations by fast computing machines, The Journal of Chemical Physics, 21(6), (1953), 1087-1092.

[33] Ng, H.K.T., Parameter estimation for a modified Weibull distribution for progressively type-II censored samples, IEEE Transactions on Reliability 54, (2005), pp. 374-380.

[34] Xia, Z.P., Yu, J.Y., Cheng, L.D., Liu, L.F., Wang, W.M., Study on the breaking strength of jute fibers using modified Weibull distribution, Composites Part A: Applied Science and Manufacturing, 40(1), (2009), 54-59.

\section{Creative Commons Attribution License 4.0 (Attribution 4.0 International, CC BY 4.0)}

This article is published under the terms of the Creative Commons Attribution License 4.0 https://creativecommons.org/licenses/by/4.0/deed.en_US 